\title{
Mobile Agent Based Distributed Network Architecture with Map Reduce Programming Model
}

\author{
Benard O. Osero ${ }^{1, *}$, Elisha Abade ${ }^{2}$, Stephen Mburu $^{2}$ \\ ${ }^{1}$ Department of Computer Science, Chuka University, Kenya \\ ${ }^{2}$ School of Computing and Informatics, University of Nairobi, Kenya \\ Received September 9, 2019; Revised October 9, 2019; Accepted October 23, 2019
}

Copyright $\odot 2019$ by authors, all rights reserved. Authors agree that this article remains permanently open access under the terms of the Creative Commons Attribution License 4.0 International License

\begin{abstract}
In the recent years, the demand for data processing has been on the rise prompting researchers to investigate new ways of managing data. Our research delves into the emerging trends of data management methods, one of which is the agent based techniques and the active disk technology and also the use of Map-reduce functions in unstructured data management. Motivated by this new trend, our architecture employs mobile agents technology to develop an open source framework called SPADE to implement a simulation platform called SABSA. The architecture in this research compares the performance of four network storage architectures: Store and forward processes(SAF), Object Storage Devices(OSD), Mobile agent with a Domain Controller (DMC) enhanced with map-reduce function and Mobile agent with a Domain Controller and child DMC enhanced with Map-reduce (ABMR): both handling sorted and unsorted metadata. In order to accurately establish the performance improvements in the new hybrid agent based models and map-reduce functions, an analytic simulation model on which experiments based on the identified storage architectures were performed was developed and then analytical data and graphs were generated. The results indicated that all the agents based storage architectures minimize latencies by up to $45 \%$ and reduce access time by up to $21 \%$ compared to SAF and OSD.
\end{abstract}

Keywords Domain Controller, Object Storage, Agent Based Storage, Metadata, Network Attached Storage

\section{Introduction}

Virtualization is a critical determinant which defines the path distributed storage array systems should follow in order to succeed. By its nature, virtualization manages data right from its source. The storage value has been changing from drives to the array cluster controllers while enhanced and data protection policies are included in such systems with time. [64].

\section{Overview of the Existing Systems}

\subsection{Direct Attached Storage}

In their classification model [4] refer this model as Direct attached storage while [54] referred this type of storage as store and forward (SAF); in this type of storage the network disks involved have to keep a copy of another redundant disk in the server. Every time a client requests for a file a copy of the file has to be kept before it is forwarded to the client for downloading. [54] further compared SAD and NASD and demonstrated that by keeping a copy of the disk there was a penalty on performance and scalability he further demonstrated an improved security mechanisms using tokenization on these platforms. He concluded that such systems can be improved by use of Object storage management schemes and he proposed further work on mobile agent and mobile code migration on a distributed network.

\subsection{Network-Attached Storage Devices}

Network-attached storage (NAS) happens at the file-level where one or more dedicated servers and disks store data and share it 1 other clients on a network.

Network-Attached Secure Disks (NASD), is a Networked object based shared storage system shown [4] in their classification taxonomy, that modifies the interface for the common direct attached storage devices and thus eliminating the server resources required for the movement of data. Figure 1 outlines the major components of NASD ARCHTECTURE [23,54].

The model allows for the transfer data directly from the 
client to the server via the file manager. The NASD execution sequence shows the requests needed until the file is downloaded to the client as explained [54-55].

The architecture below demonstrates a framework of the NASD Architecture by [54-55].

\subsection{Object-Based Storage (OSD)}

For Object storage the data is broken into small connected units called objects kept in a single repository (pool), instead of being kept as blocks on servers.

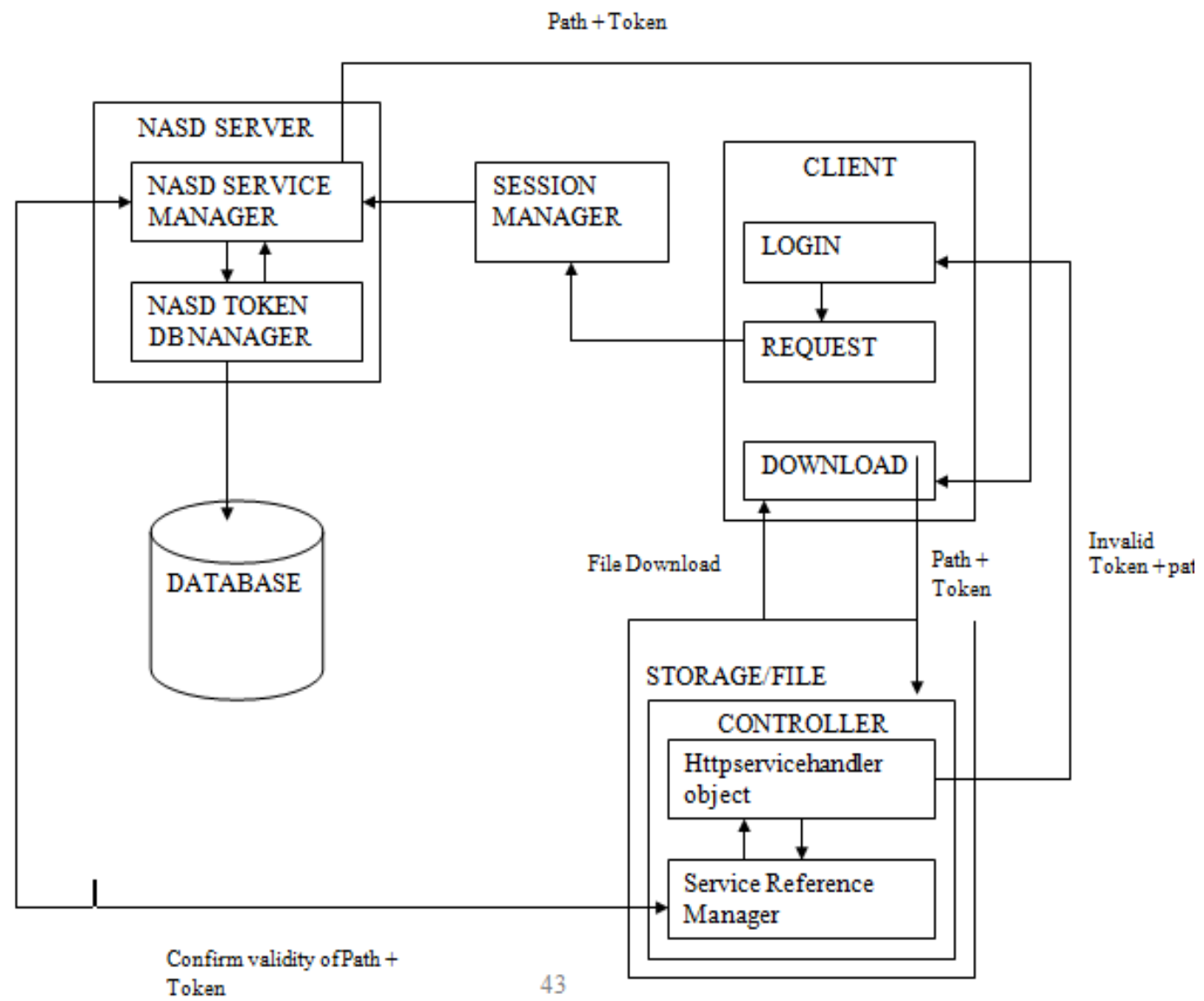

Figure 1. The major components of NASD Architecture

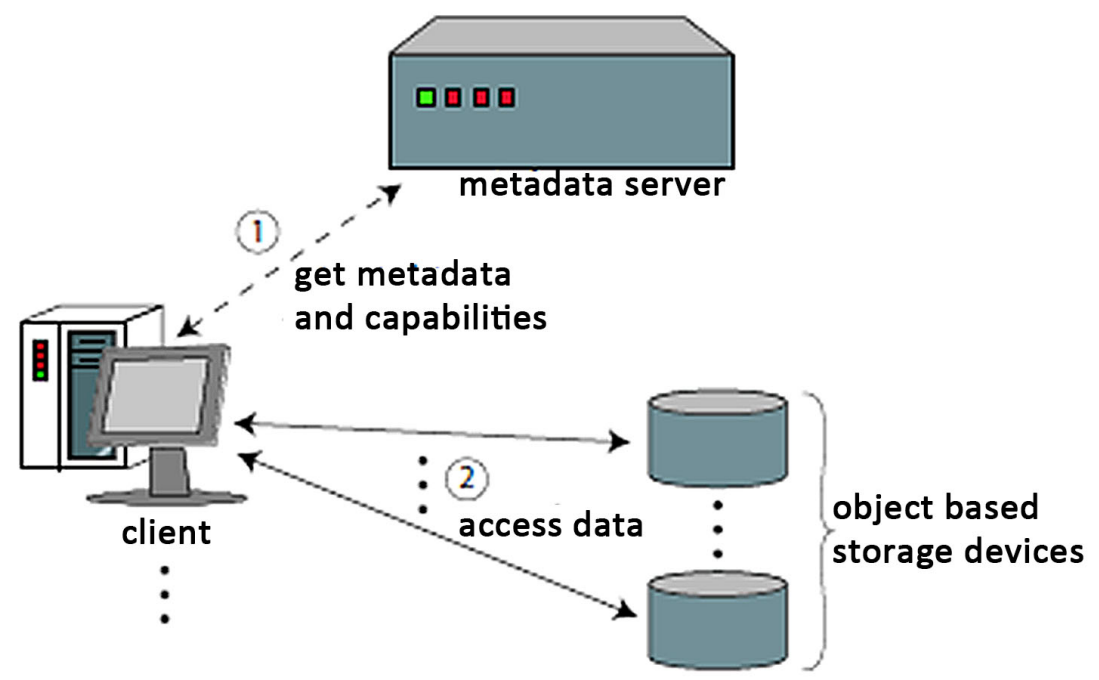

Figure 2. Direct client access 
Figure 2 above depicts how client interaction between the server in order to get metadata access information [29]. The Object storage concept builds on previous work on NASD researched $[23,54,55]$.

A storage object may be described as a sequence of addressed bytes, including other associated features accessed via a file interface with read, write and delete commands. This model is limited by the available bandwidth from a file server, clients and connected storage.

Object-based storage Research has been an ongoing subject that continues to elicit a lot of interest from researchers [17,45,18] early products such as Lustre [53], SWIFT [51] Panasas in [59] and [62] have equally appeared in the market. Additionally, object-based storage is being leveraged as a means to put together application-defined and other data features for compliance purposes [12,62]. For object-based storage to be of better use outside its current applications, it has to handle little chunks of files efficiently.

\subsection{Deficiencies with the Current Systems}

i) There has been an increase in the data processing demands and this requires faster systems that can scale well over short periods of time, most of the systems so far covered in the literature are either traditional in nature like SAF (DAS) systems which are array based or they are object based like NASD and OSD but they experience high latencies and decreased throughput as witnessed in $[55,54,26,17,45,18]$. ii) Low bandwidth and unmanaged Latencies have a major effect on the performance a distributed cluster or network, data prefetching methods have been implemented through predictive prefetching algorithms, but little progress has so far been made on metadata management schemes [86]; Although, [7] provide a solution to bandwidth issues which occur when the client and server interact, this solution only improves on bandwidth and not latencies.

Based on related works discussed above, there are major gaps or potential improvement areas in the approaches in the design and implementation of a distributed network and ways of testing performance of these systems. This research therefore dedicates its effort towards development and testing of concepts, components and implementation of suitable algorithms and models required for implementation of a suitable architectural model for distributed storage on a network.

\section{Proposed Solution}

The system to solve the above identified gaps introduces an intelligent way of managing a distributed network through the use of mobile agents, the agents are controlled by a central controller called the Domain Controller that keeps track of all the mobile agents in its registry. This intelligent network has the capability of minimizing latencies by localizing data in its local cache for subsequent client access.

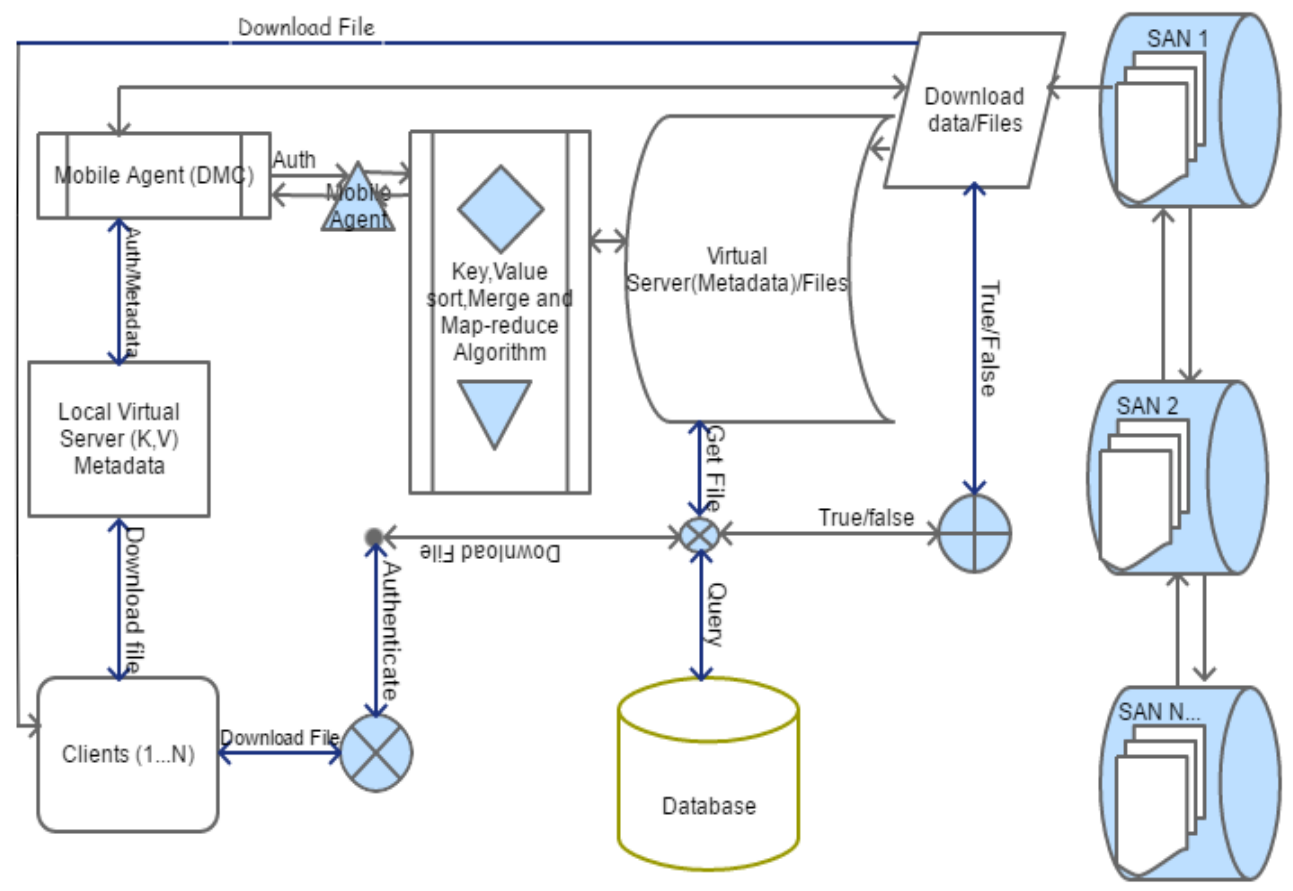

Figure 3. A Conceptual Architecture for intelligent objects using agents and Map-reduce. 
Figure 3 above shows an architectural model of the agent based design using map-reduce, it is a three tiered model with the client as the front end the virtual server as the middle tier and storage SAN as the backend, it also includes the following functionalities:

Map-reduce Functions represented as shown below:

$$
\left[\left(k_{i}, v_{i}\right), \ldots,\left(k_{n}, v_{n}\right)\right]: \forall i=1 \ldots n:\left(\mathrm{k}_{\mathrm{i}}, \in \mathrm{K}, v_{\mathrm{i}}, \in \mathrm{V}\right)
$$

$\mathrm{k}_{i}=\mathrm{i}^{\text {th }}$ input and $\mathrm{v}_{i}=\mathrm{i}^{\text {th }}$ input value. $\mathrm{K}$ represents the key domain and $\mathrm{V}$ represents the value domains. Map reduce function performs the responsibility of splitting the key, value input values to subsets and eventually distributing them to respective nodes. The mapping can be obtained as follows

$$
\begin{gathered}
\text { Map: } \mathrm{K} \times \mathrm{V} \rightarrow(\mathrm{L} \times \mathrm{W})^{*} \\
(\mathrm{k}, \mathrm{v}) \rightarrow\left[\left(\mathrm{l}_{1}, \mathrm{x}_{\mathrm{l}}\right), \ldots,\left(\mathrm{l}_{\mathrm{l}}, \mathrm{x}_{r}\right)\right]
\end{gathered}
$$

$\mathrm{L}$ and $\mathrm{W}$ represents the key and value domains indicating the key-value input pairs: $(\mathrm{k}, \mathrm{v})$ mapped onto many key and value pairs: $\left.\left(1_{1}, \mathrm{x}_{1}\right), \ldots,\left(\mathrm{l}_{1}, \mathrm{x}_{r}\right)\right]$. The reduce phase is as follows:

$$
\begin{gathered}
\text { Reduce: } \mathrm{L} \times \mathrm{W}^{*} \rightarrow \mathrm{W}^{*} \\
\left(1,\left[\mathrm{y}_{1}, \ldots, \mathrm{y}_{s l}\right]\right) \rightarrow\left[W_{1}, \ldots, W_{m l}\right]
\end{gathered}
$$

Keys with similar results are grouped together in this phase, input may have dissimilar values, the output generated has same keys. This requires sorting of results and consolidating them for final processing. This is performed by input function $(\mathrm{L} \times \mathrm{W}){ }^{*}$ which when processed produces $(\mathrm{L} \times \mathrm{W}){ }^{*}$ as sorted input values for the reduce function. $(\mathrm{L} \times \mathrm{W}){ }^{*}$ can also be mapped to generate a new list $\mathrm{W}^{*}$. Map reduce can generally be summarized as follows:

$$
\text { Map Reduce: }(\mathrm{K} \times \mathrm{V})^{*} \rightarrow(\mathrm{L} \times \mathrm{W})^{*}
$$

This function is responsible for sorting and reducing metadata functions which can then be transported to client side for further processing.

\subsection{Solution Outline}

The Storage virtualisation architecture in this research involves a logical access to storage resources through defined metadata rather than having access to the physical resource itself; because physical file occupies more space. In the storage unstructured files are not necessarily arranged and accessed in a certain predefined sequence; this is made much more difficult when the data is randomly assorted into the storage from random sources; since their metadata is randomly placed in the storage searching for a file requested by the client becomes very difficult. Therefore, several methods or parameters can be employed to shuffle or sort the metadata depending on the intended mapping outcome i.e. one can sort as per the number of occurrences of the file, the IP address similarities, the file Index name or Number similarities etc.
To address the gaps in this research which have a direct impact on both latencies and response times the conceptual Architecture defined in Figure 3 employed metadata sorting and shuffling by use of map reduce algorithm by creating corresponding IP addresses of the respective client domains which were then mapped to a mobile agent for migration to a Domain Controller (DMC), where they were executed henceforth, a client can terminate normally or abnormally in case of an unrecoverable event.

The main objective of this research was to test whether a mobile agent can improve the performance of a distributed network if used together with the map reduce algorithm to sort the metadata within the virtual server in order to create sorted IP domain localities. A conceptual architecture was introduced and discussed in detail in figure 3. However, the conceptual architecture being a high level design model; only identified key architectural artifacts/components that of a three tiered distributed storage architecture.

In order to actualize the concepts in this research into a concrete design and develop a model capable of giving indicative performance, some important issues needed to be tackled as follows:

i) To develop and implement a simulator that will assist in testing the scalability and performance.

ii) To implement a search, sort and mapping mechanism using map-reduce functions that can improve in searching, sorting and mapping of metadata in the virtual server.

iii) To Implement Agents on a Distributed network using the SPADE architecture.

iv) Evaluate the performance of Agent based Distributed system using Map-reduce with non-agent based distributed network environment specifically SAF and OSD in the implemented simulator.

\subsection{The Need for the SABSA Simulator}

Our simulation model utilized the experimental methodology in order to answer various research questions raised by the researcher. The simulation model used in this research was developed from scratch using open source software tools; this was arrived at after critical analysis of various simulation platforms. the Network simulation models so far existing are only meant for the first three Physical layers of the OSI model (Physical, Datalink and the Network) and therefore, they were suited for the physical characteristics of the network, but our research could not be supported by this popular network platforms as described by [71] and [31] because we were performing file simulation at the sockets level; which occurs at the upper layers of the OSI model (Transport and Session layers)and therefore there was need to design a simulator to cater for the desired and unique file simulation features.

Our simulation model was meant to test performance improvement after introducing agents into the existing SAF and OSD models and also sorting metadata using map 
reduce approach. The performance measures have been described by [25,90,50] and [85] which were adopted as a performance measure for our SABSA engine to provide the metrics that were used for this research.

\subsubsection{Simulator Requirements and Design}

Our simulator was developed using standard Python APIs for the design of the client and server machines using Sockets and standard Docker Containers running on the Linux OS cluster for file storage virtualization (SAN) and also SHA1 encryption scheme for security.

\subsubsection{System Requirements and Configuration}

The system was configured to run on an AWS EC2 t2. micro virtual machine instance with:

The host machine was a HP Folio 9470, equipped with an Intel Core(TM)i7 CPU $2.60 \mathrm{GHz}$ CPU, 8 GB RAM, a 500 GB hard disk drive, 64-bit OS, x64 based processor and a GSM wireless network connection and a Windows 2015 Pro Operating System. The system was also hosted on the Amazon EC2.

The system also required installation of Docker on the virtual machine to run it. Configuration was done using Docker files which specified the VM image used as well as run instructions for system setup. Instructions included setting environment variables that were used by the application and installing of the packages that were used as well as exposing ports which were to be accessed from outside of the virtual machine.

\section{Simulations and Results}

The simulator in this research was developed using standard Python APIs for the design of the client and server machines using Sockets and standard Docker Containers running on the Linux OS cluster for file storage virtualization (SAN) and also SHA1 encryption scheme for security. Our Model used a Simulation model with various experiments being carried out at various levels.

\subsection{Simulation Model}

The Simulation and experimental methodologies were employed for our research as described by [89] and [8]. Our model development and testing followed the three basic steps defined in the literature by [8] as follows:

i) Created an Architectural model for approximating the events.

ii) The model was then simulated in a computer software, which allowed for the repeated observation of the model. After one or many simulations of our model iii) A third step was analysis. Analysis aided in the drawing of conclusions, verification and validating the research, and hence enabling us to make recommendations based on various iterations or simulations of the model.

The simulation model utilized the experimental methodology in order to answer various research questions raised by the researcher and also described by [52] in developing his research methodology. The simulation model used in this research was developed from scratch using open source software tools; this was arrived at after critical analysis of various simulation platforms of the Network simulation models so far existing are only meant for the first three Physical layers of the OSI model (Physical, Datalink and the Network) and therefore, were suited for the physical characteristics of the network, but our research could not be supported by this popular network platforms as described by [71] and [31] because we were performing file level simulation at the sockets level; which takes place at the upper layers of the OSI model (Transport and Session layers)and therefore there was need to design a simulator to cater for the desired and unique file simulation features.

Our simulation model was meant to test performance improvement after introducing agents into the existing SAF and OSD models and also sorting metadata using map reduce approach. The performance measures have been described by $[25,90,50]$ and [85] which were adopted as a performance measure for our SABSA engine to provide the metrics that were used for this research.

\subsection{The Simulation Procedure}

To easily address the gaps in this research a simulation testbed referred to as SABSA (Secure Agent Based Architecture) was developed in phases as follows:

1. Phase 1: Design and implementation a direct file access.

2. Phase 2: Design and Implementation of Object storage device (OSD).

3. Phase 3: Mobile agent Domain Controller (DMC) enhanced with map-reduce function.

4. Phase 4: Mobile agent based Domain Controller with child DMC controllers enhanced with Map-reduce.

5. Repeat Phase 2, 3 and 4 with sorted metadata.

The results were then presented in regard to the following identified parameters: Latencies, throughput and Scalability; either with sorted or unsorted metadata.

The existing files (Bytes) were first classified in the following table before they were run in the simulator: 


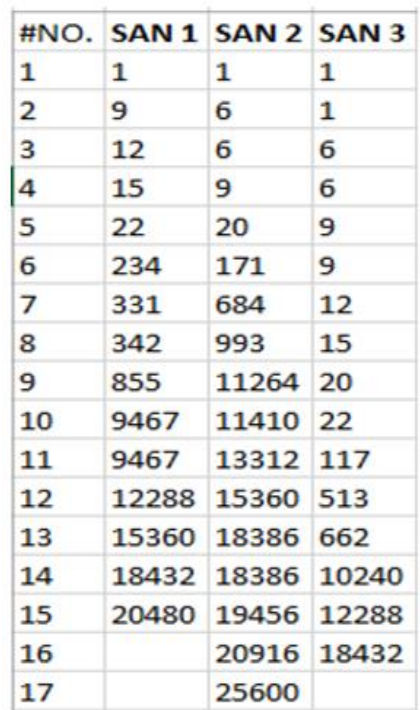

The above files were extracted from the SAN container (SAN 1, SAN2 and SAN 3) and then entered into excel sheet and sorted in ascending order and the files were then sorted according to the combinations of three sizes as demonstrated in table 2 below.The file sizes were capped at 30000 bytes; because files beyond this size slowed down the machine drastistially.

4.2.1. File Classifications in Bytes (1 File Per San).

Table 2. Workload distribution matrix

\begin{tabular}{|c|c|c|c|c|}
\hline SAN 1 & SAN 2 & SAN 3 & RANDOMLY SELECTED FILE OPTIONS & CASE DISCUSSION \\
\hline SMALL & SMALL & SMALL & & CASE 1 \\
\hline SMALL & SMALL & MEDIUM & $\mathrm{x}$ & \\
\hline SMALL & SMALL & LARGE & $\mathrm{x}$ & \\
\hline SMALL & MEDIUM & SMALL & & CASE 2 \\
\hline SMALL & MEDIUM & MEDIUM & $\mathrm{x}$ & \\
\hline SMALL & MEDIUM & LARGE & $\mathrm{x}$ & \\
\hline SMALL & LARGE & SMALL & & CASE 3 \\
\hline SMALL & LARGE & MEDIUM & $\mathrm{x}$ & \\
\hline SMALL & LARGE & LARGE & $\mathrm{x}$ & \\
\hline MEDIUM & SMALL & SMALL & $\mathrm{x}$ & \\
\hline MEDIUM & SMALL & MEDIUM & $\mathrm{x}$ & \\
\hline MEDIUM & MEDIUM & MEDIUM & & CASE 4 \\
\hline MEDIUM & MEDIUM & LARGE & $\mathrm{x}$ & \\
\hline MEDIUM & LARGE & SMALL & $\mathrm{x}$ & \\
\hline MEDIUM & LARGE & MEDIUM & & CASE 5 \\
\hline MEDIUM & LARGE & LARGE & $\mathrm{x}$ & \\
\hline LARGE & SMALL & SMALL & $\mathrm{x}$ & \\
\hline LARGE & SMALL & MEDIUM & $\mathrm{x}$ & \\
\hline LARGE & SMALL & LARGE & $\mathrm{x}$ & \\
\hline LARGE & MEDIUM & SMALL & $\mathrm{x}$ & \\
\hline LARGE & MEDIUM & MEDIUM & $\mathrm{x}$ & \\
\hline LARGE & MEDIUM & LARGE & $\mathrm{x}$ & \\
\hline LARGE & LARGE & SMALL & $\mathrm{x}$ & \\
\hline LARGE & LARGE & MEDIUM & $\mathrm{x}$ & \\
\hline LARGE & LARGE & LARGE & & CASE 6 \\
\hline
\end{tabular}




\section{Workload Analysis for Time, Latencies and Throughput}

Various workloads were classified as per the defined set parameters in: Table 1, then various workloads were run per each SAN as indicated in table 1: under the defined parameters a summary of various outputs were generated under various Cases were used to the analyze performance of SABSA engine under varied workloads in all the SAN containers as indicated in Tables $3-6$ below.

Table 3, 4 and 6 show Comparison graphs as per the file classification matrix

The graphs have been inserted into a table with three columns; column 1 indicated by part a) represents 100 client requests, column 2 indicated by b) represents 1000 client requests and column three represents the case numbers identified by the file classifications in table 2 . The classifications were done as either sorted or unsorted metadata functions as follows:

i) Design and implementation a direct file access-In this case there is no metadata involved, but the file is transmitted directly to the client.

ii) Design and Implementation of Object storage device (OSD)-In this case the metadata is centralized and presented as an unsorted array in the virtual server.
The metadata can further be sorted before being handled by the mobile agents which creates sorted metadata domains.

iii) Mobile agent Domain Controller (DMC) enhanced with map-reduce function-For mobile agents to function they are centrally managed by the domain controller, the agents collects the sorted metadata and moves it to the domain controller for further distribution. The unsorted metadata remains as case ii above.

iv) Mobile agent based Domain Controller with child DMC controllers enhanced with Map-reduce- Case 2 above can further be decentralized to create more child processes distributed and controlled by a centralized agent controller still referred to as the domain controller. The agents in this case can also handle both sorted and unsorted metadata objects.

\subsection{Latencies Comparison over Time}

The graph in this table section represents the changes of latencies over time and it is subdivided into two column sections a) and b): column a) represents 100 client requests and column per millisecond b) represents 1000 client requests per millisecond. 
Table 3. Latencies Comparison over time

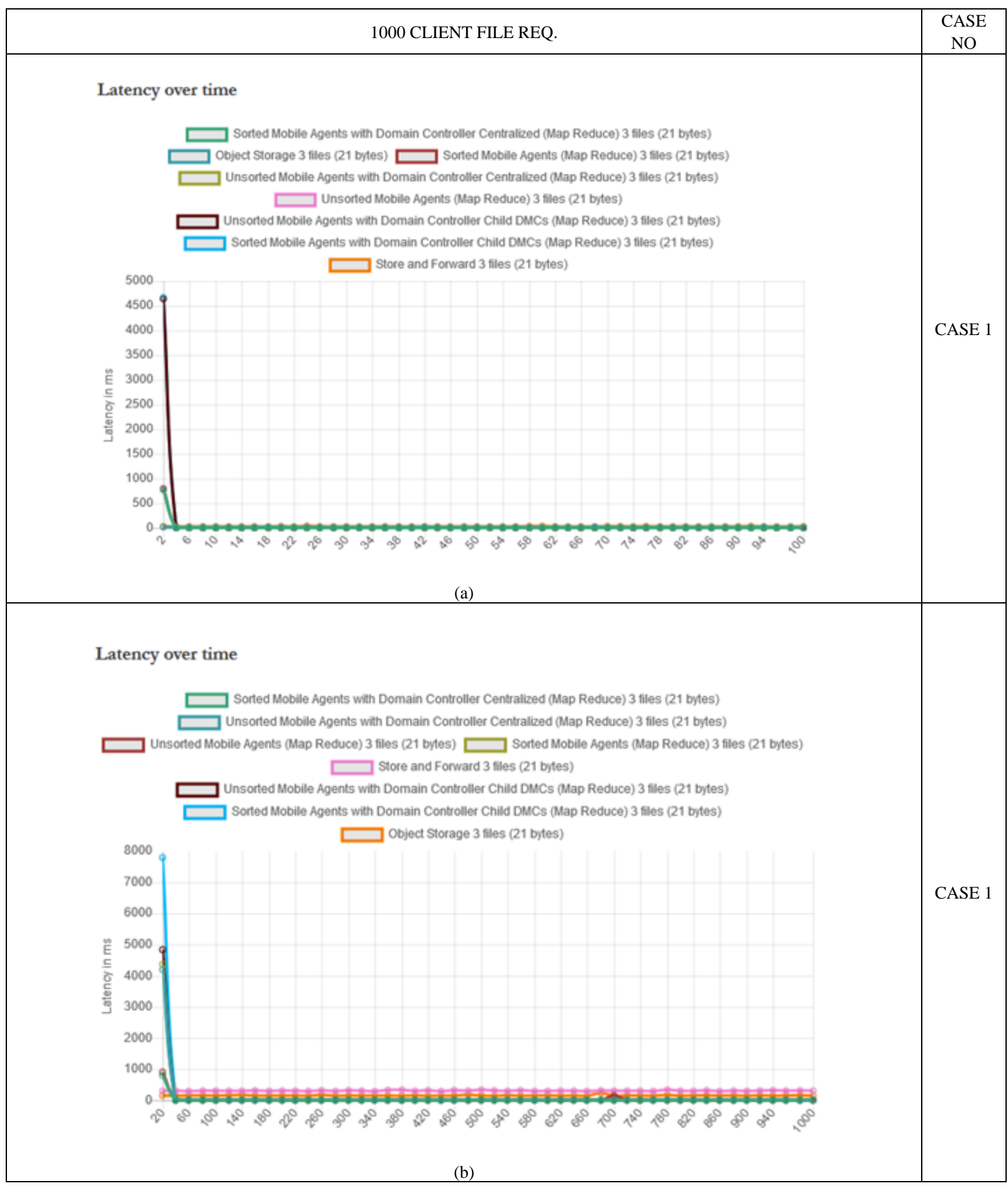


atency over time

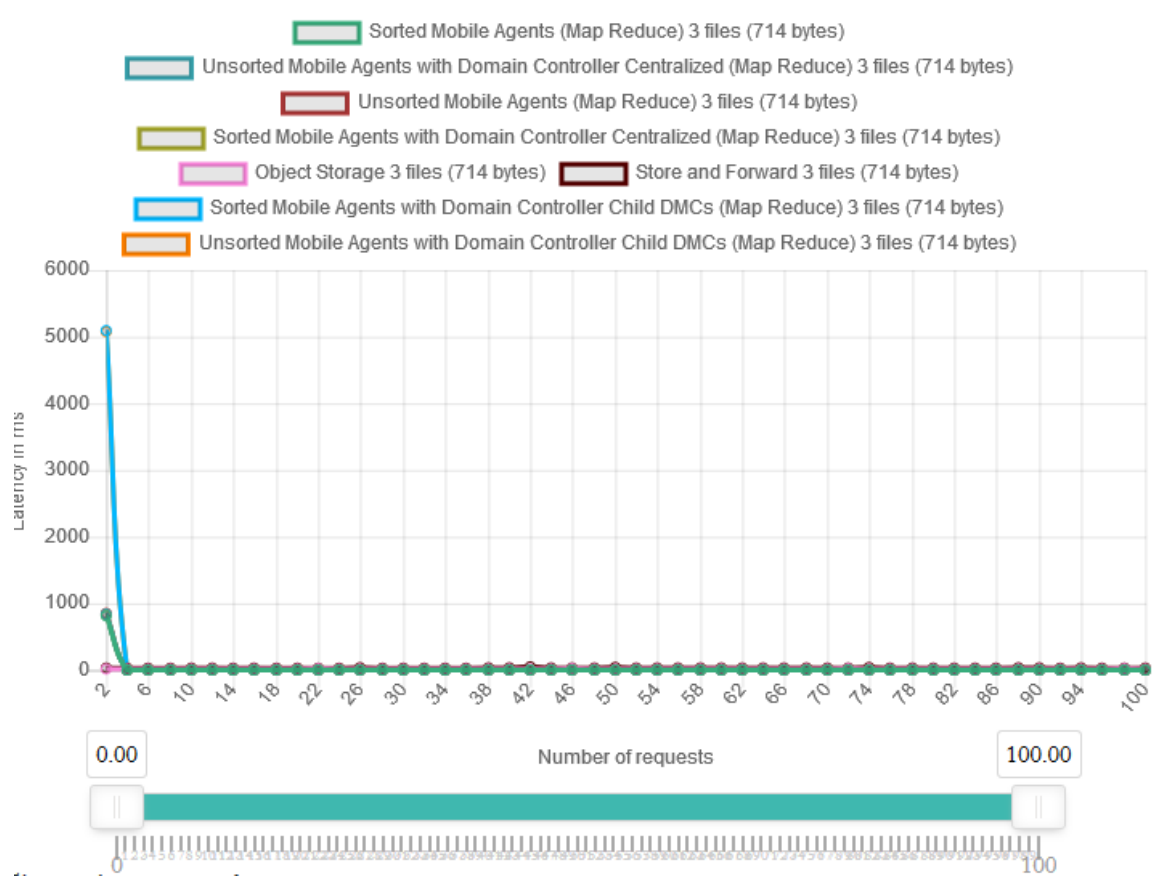

(a)

\section{Latency over time}

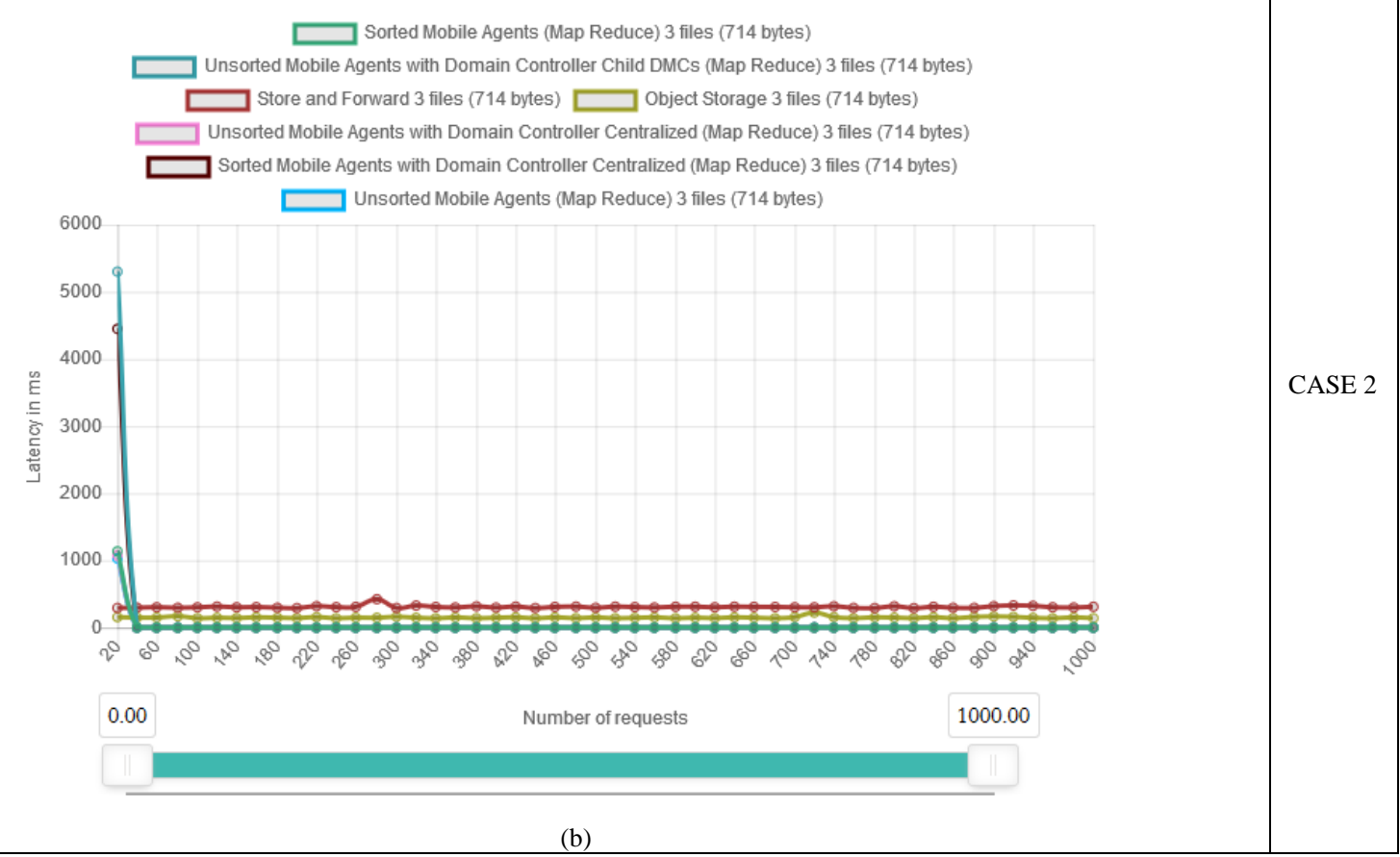




\section{Latency over time}

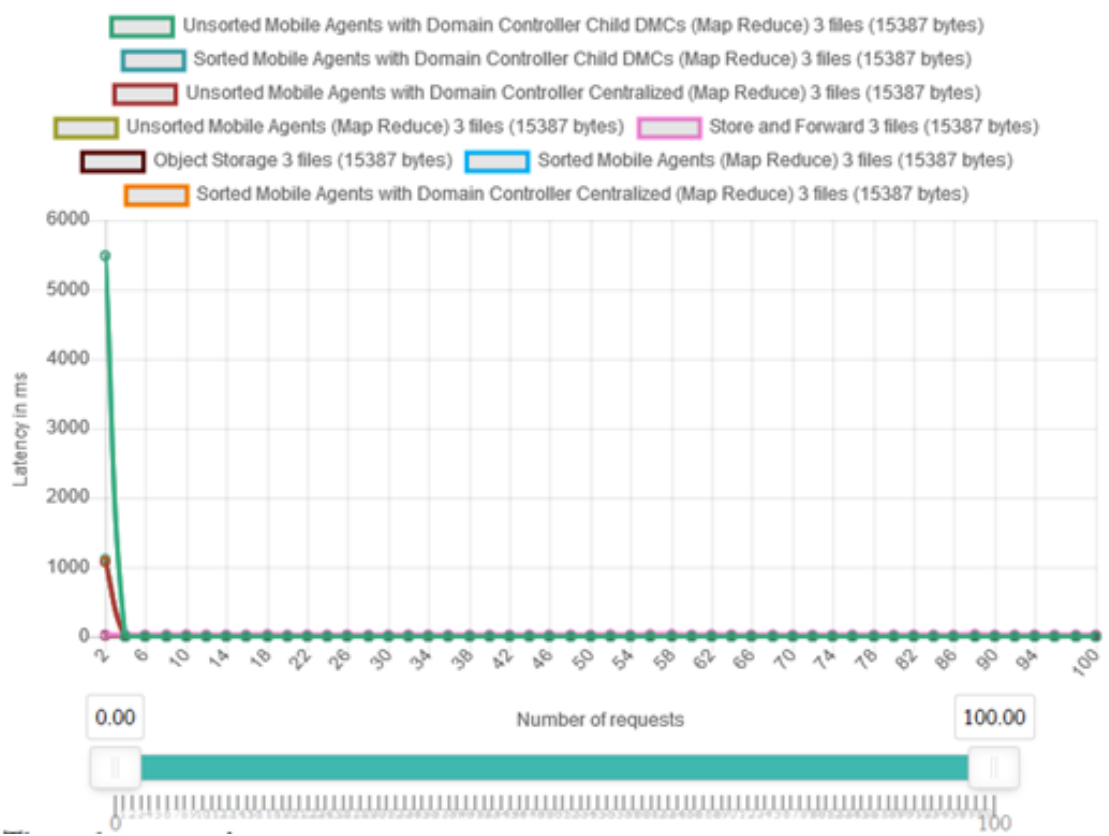

(a)

\section{Latency over time}

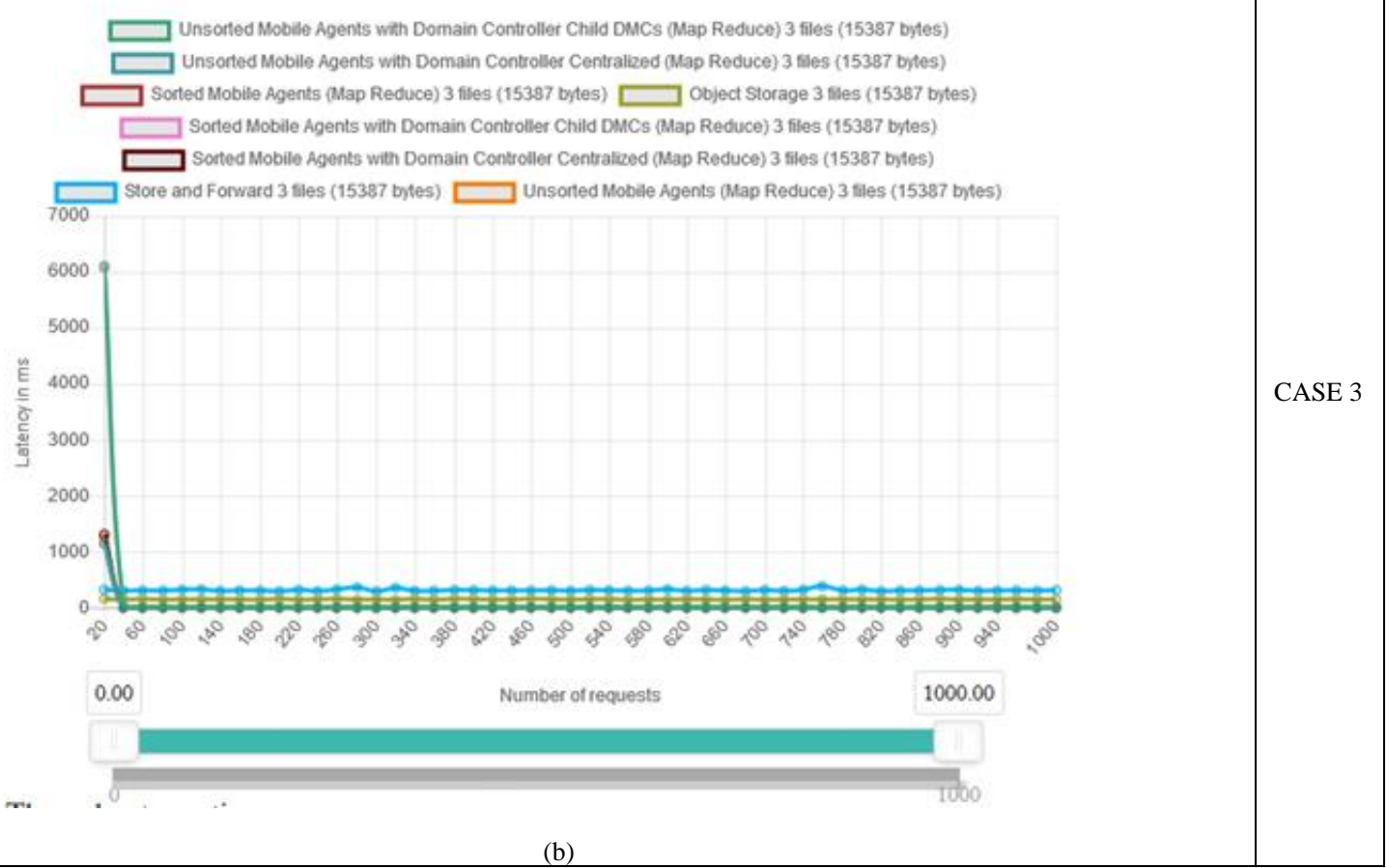




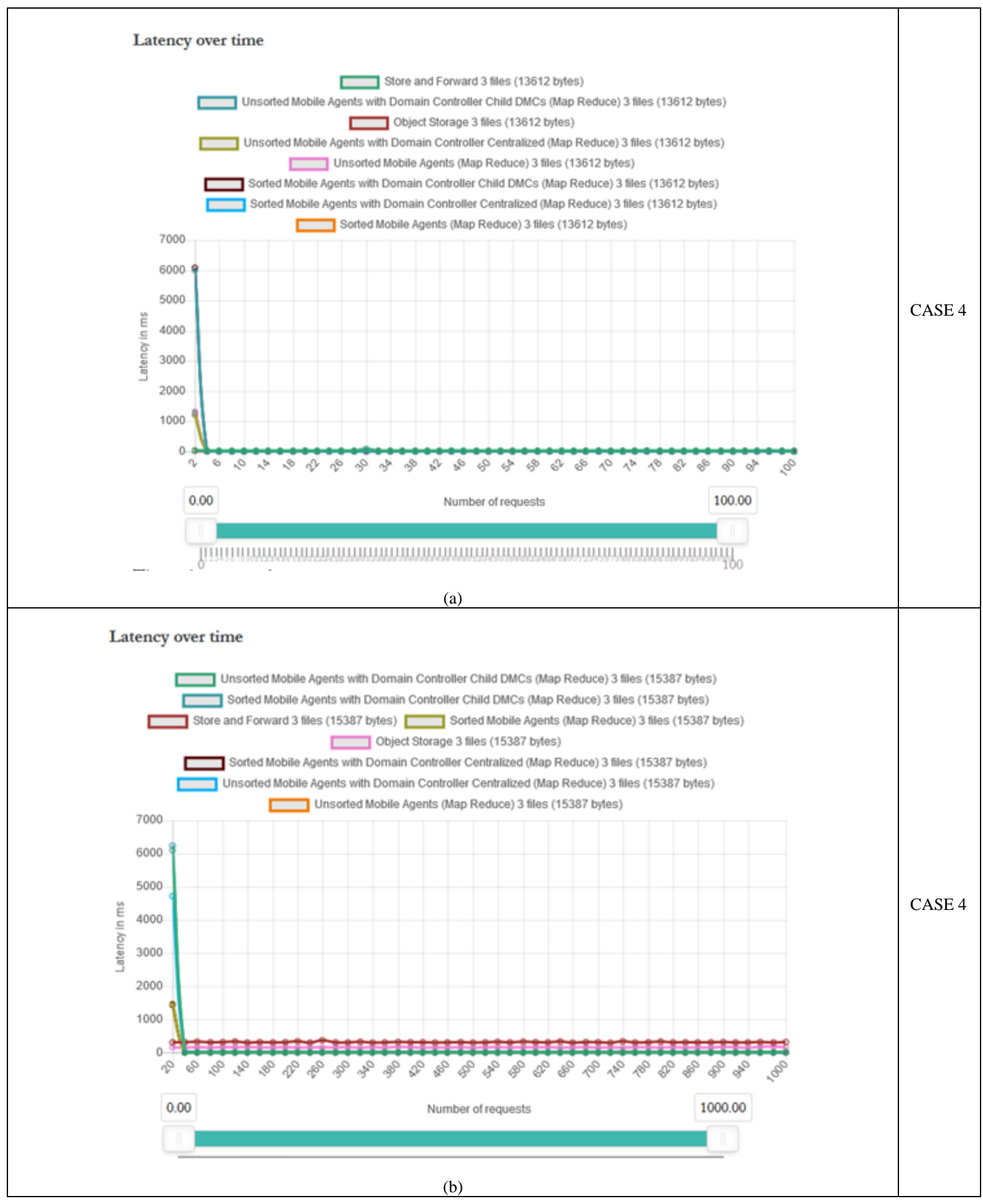

Table 3 shows comparison of various graph outputs under 100 and 1000 client classified under various CASES 1,2,4 and 6 part a) represents 100 client requests and part b) represents 1000 client requests

\subsection{Throughput over Time}

The graph in this table section represents the changes of Throughput over time and it is subdivided into two columns a) and b): column a) represents 100 client requests and column b) represents 1000 client requests. 
Table 4. Throughput over time

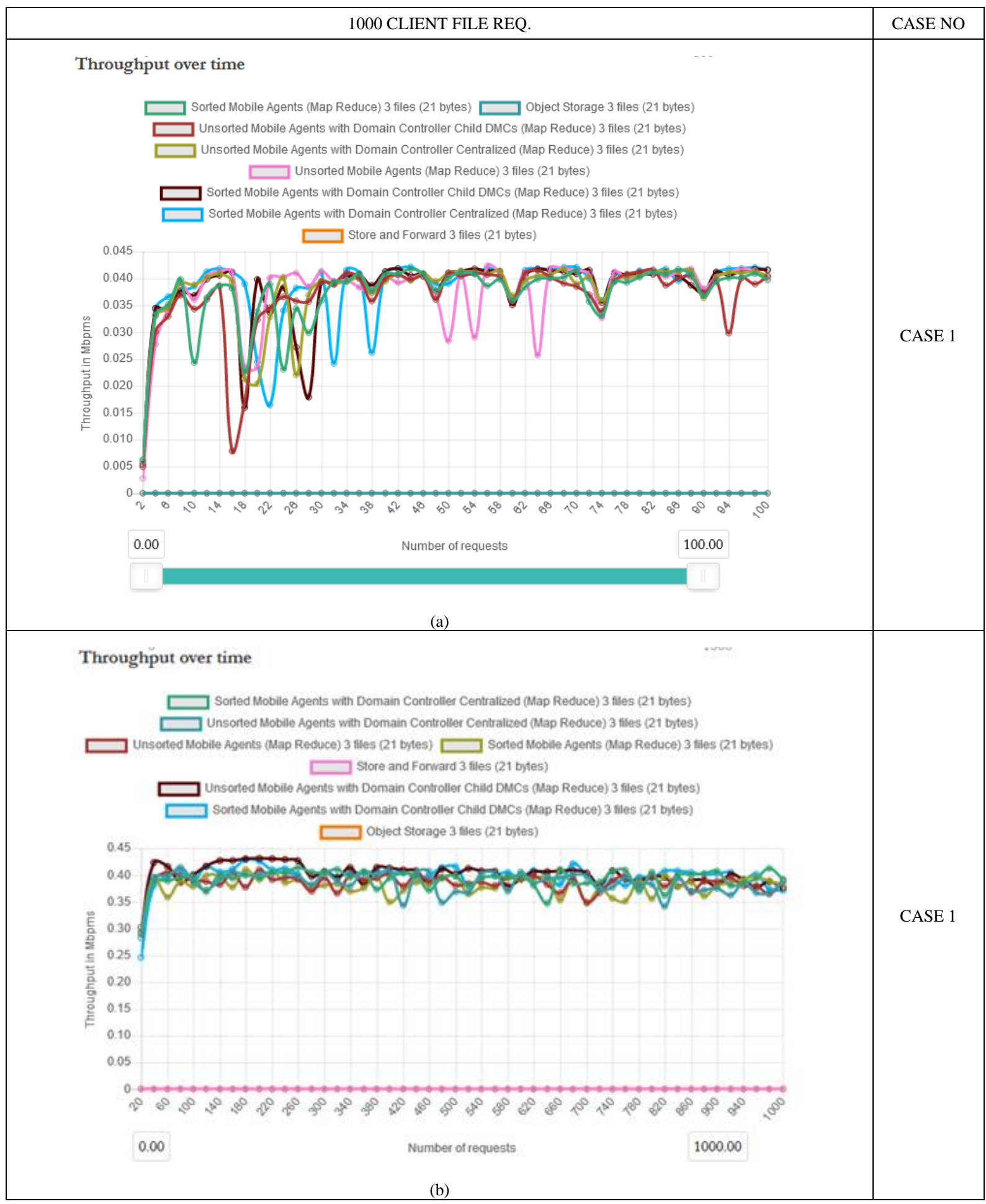




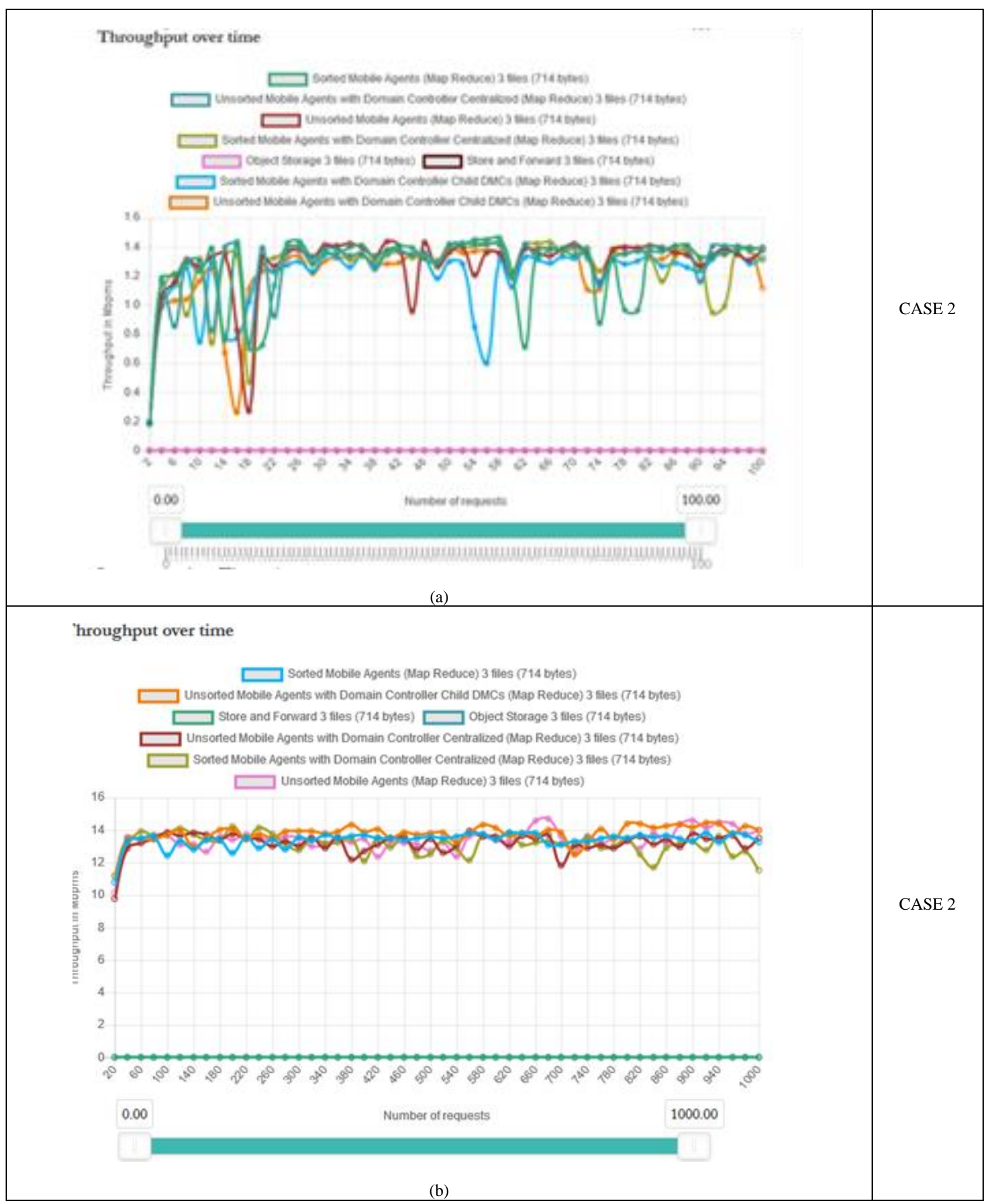


Throughput over time

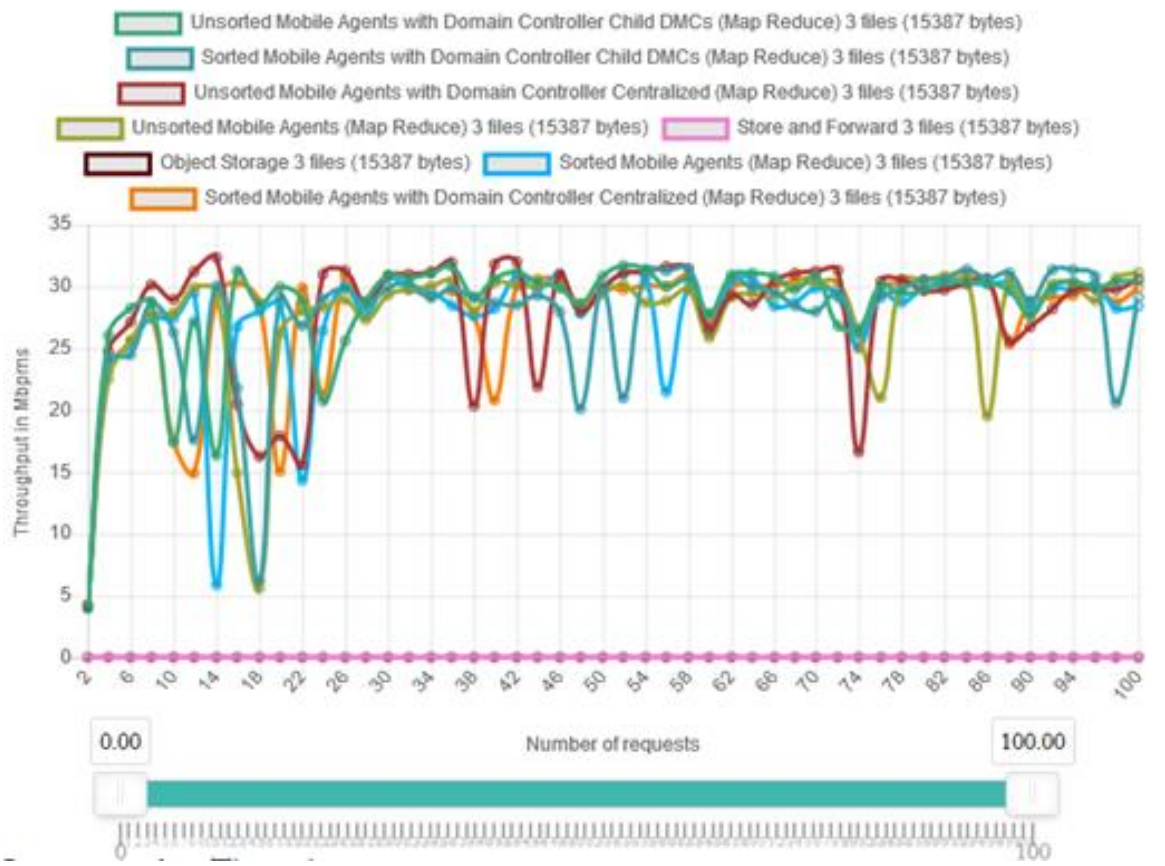

(a)

\section{Throughput over time}

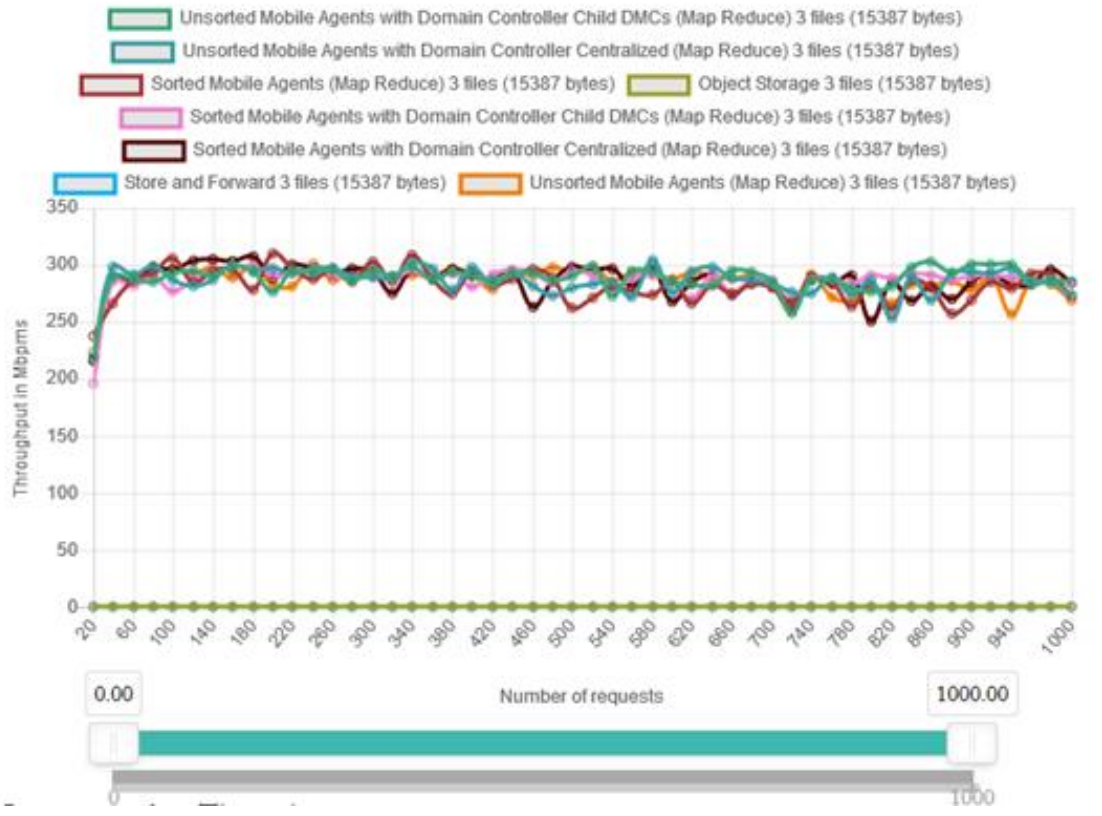




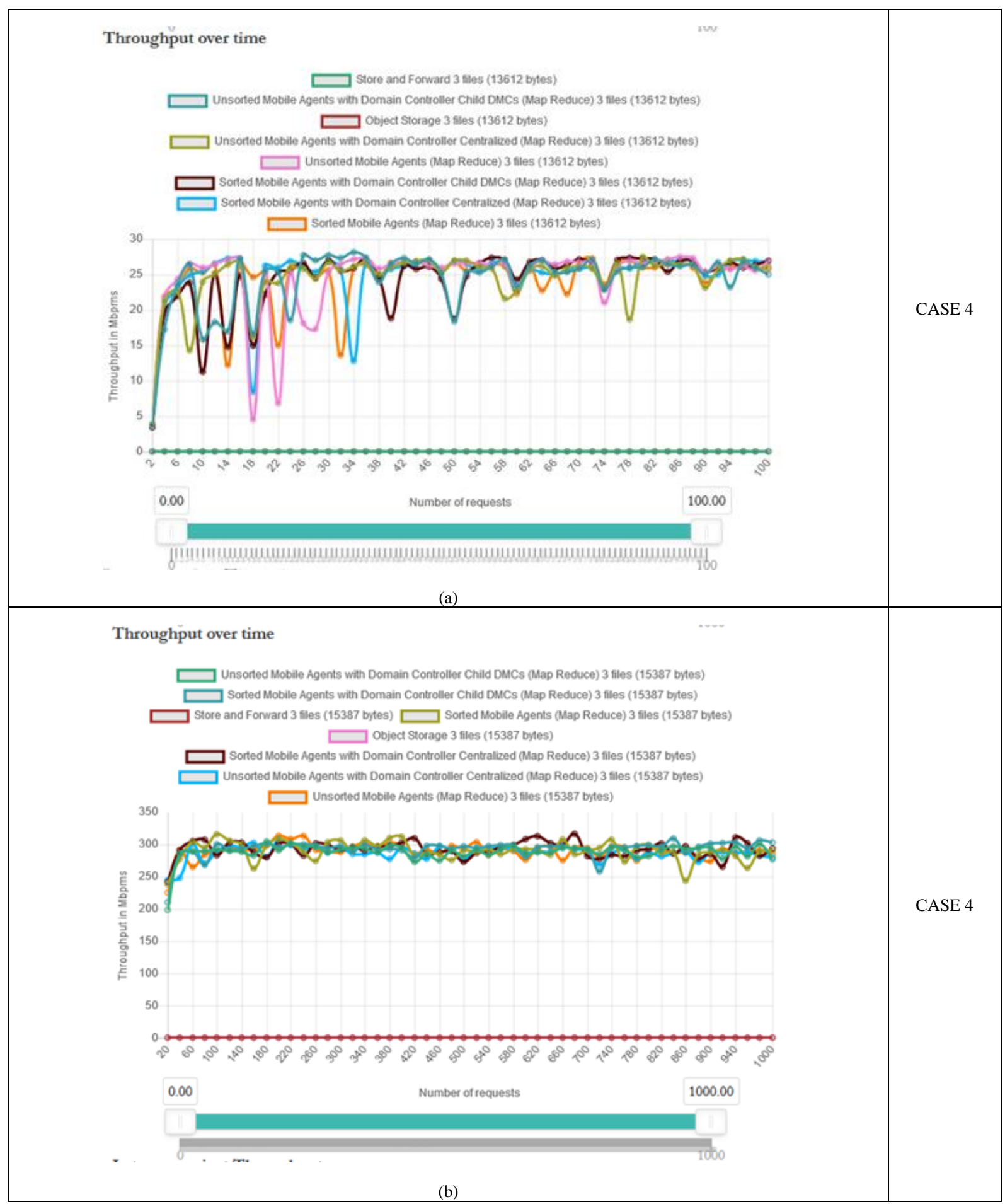

In table 4 case for instance indicates that in both case a and b store and forward has the minimum throughput at Zero and map reduce and sorting by mobile agents also has a significant improvement in throughput in all the cases observed, store and forward has a worst throughput in all cases and object based storage has an average throughput in all the cases. The maximum observed through for case $4 \mathrm{a}$ is $25 \mathrm{Mbpms}$, which drastically increases tenfold to $250 \mathrm{Mbpms}$. In all cases agents and map reduce combined show a very good improvement in throughput.

\subsection{Latency against Throughput}

The graph in this table section represents the effects of latencies on throughput and it is subdivided into two column Sections a) and b): column a represents 100 client requests and column b) represents 1000 client requests. 
Table 5. Latency against throughput

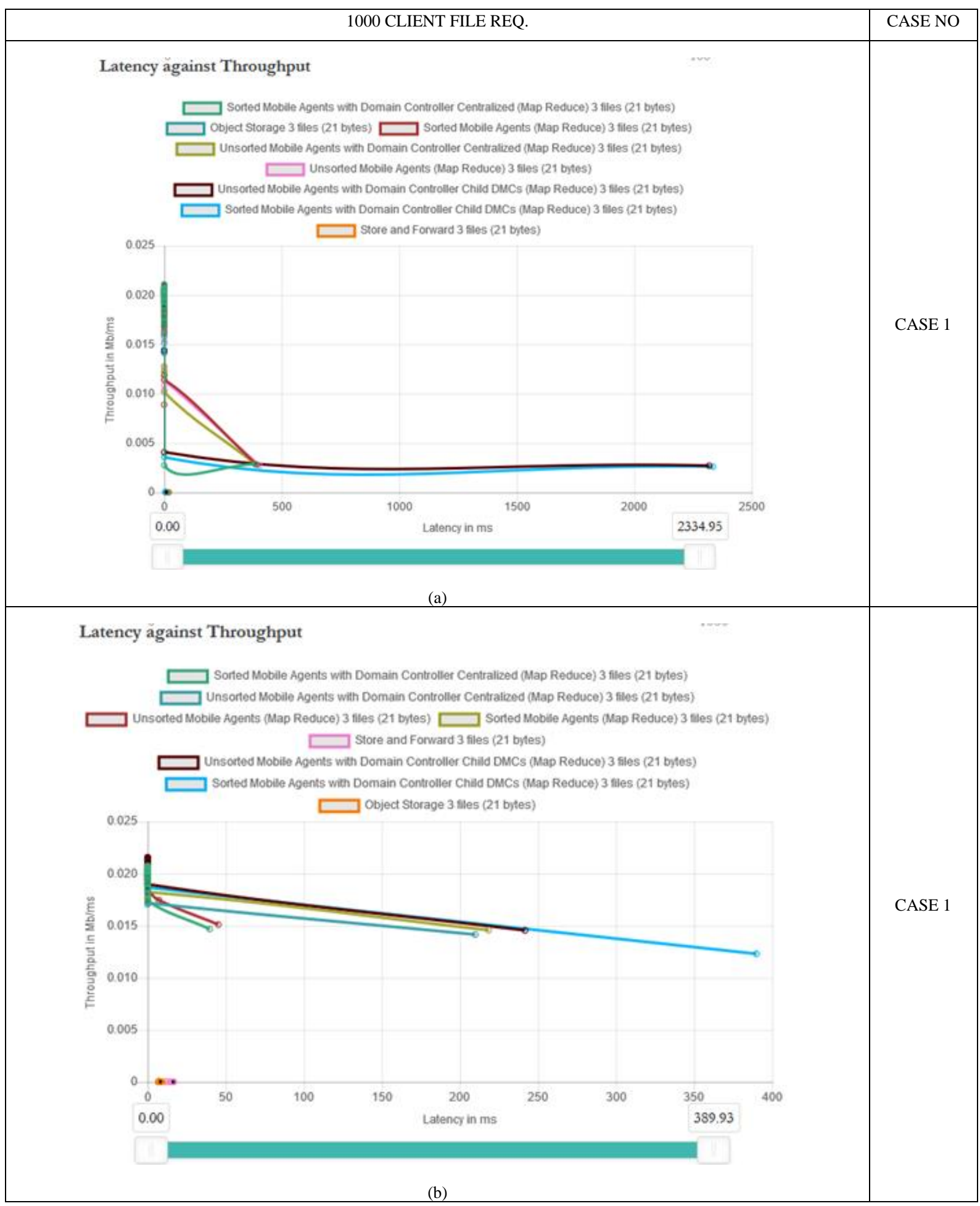


Latency ăgainst Throughput

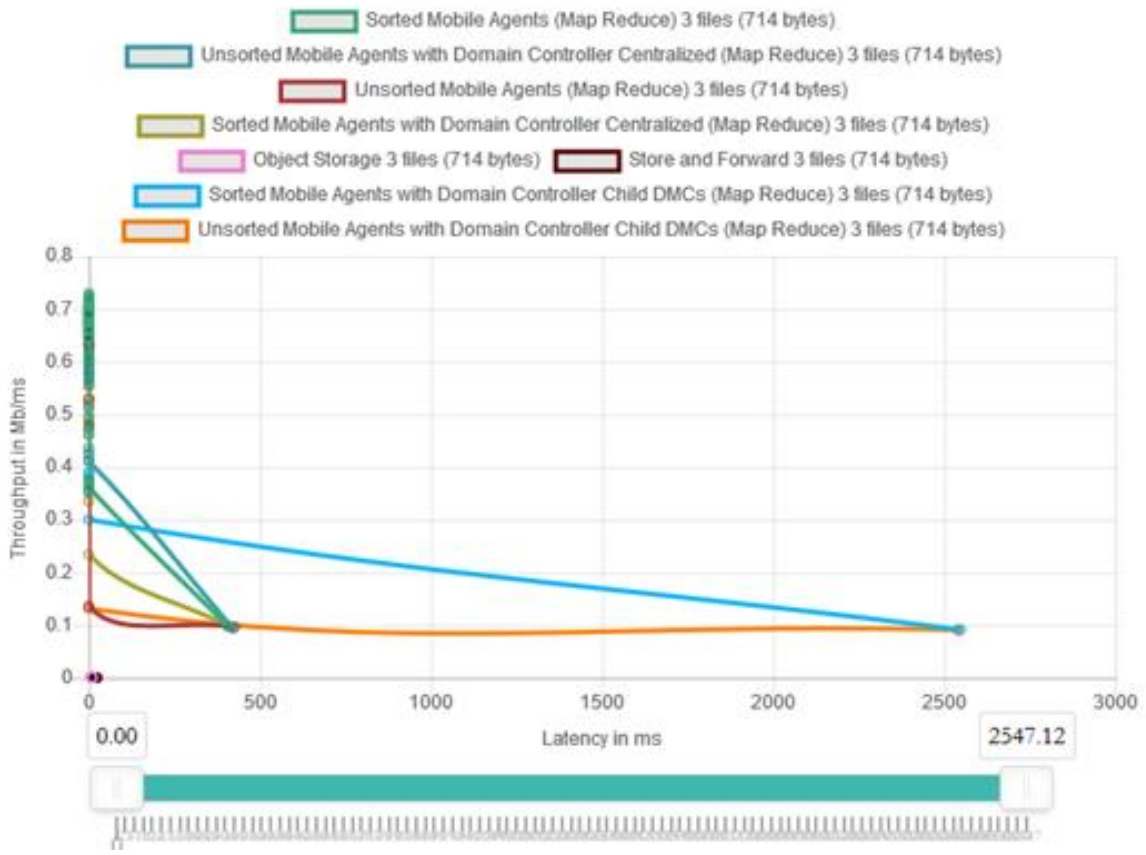

(a)

\section{Latency ägainst Throughput}

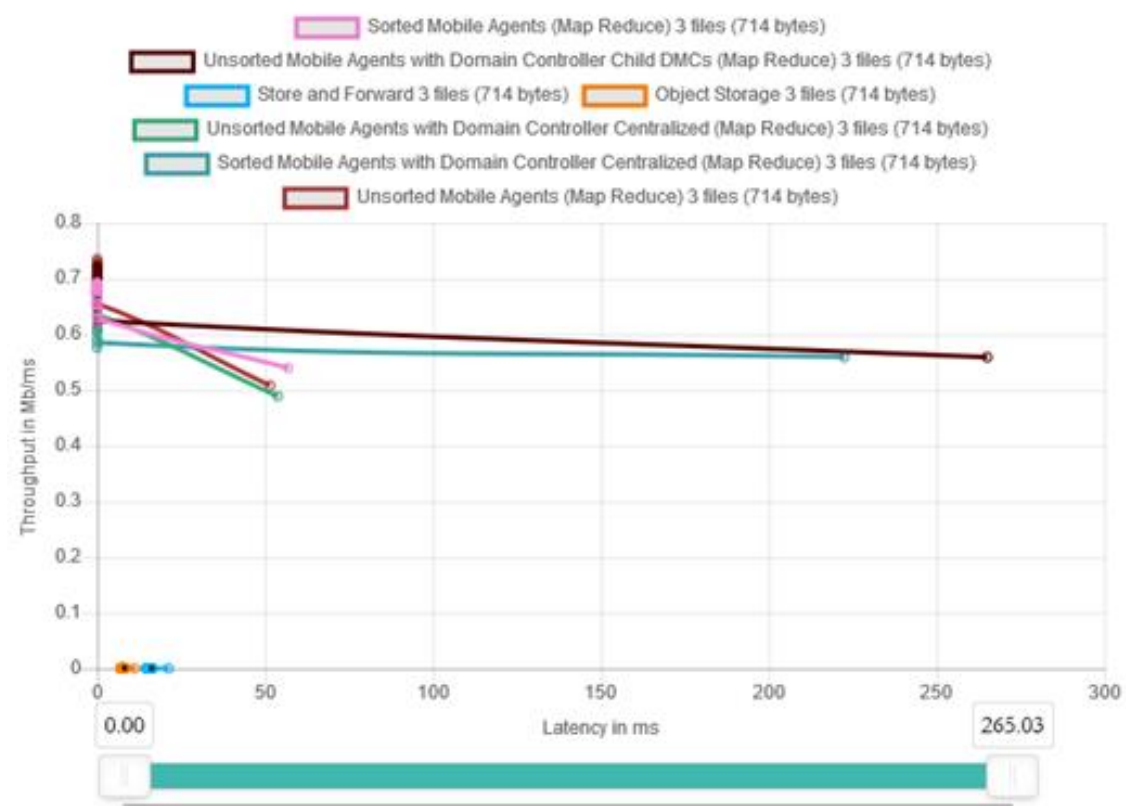




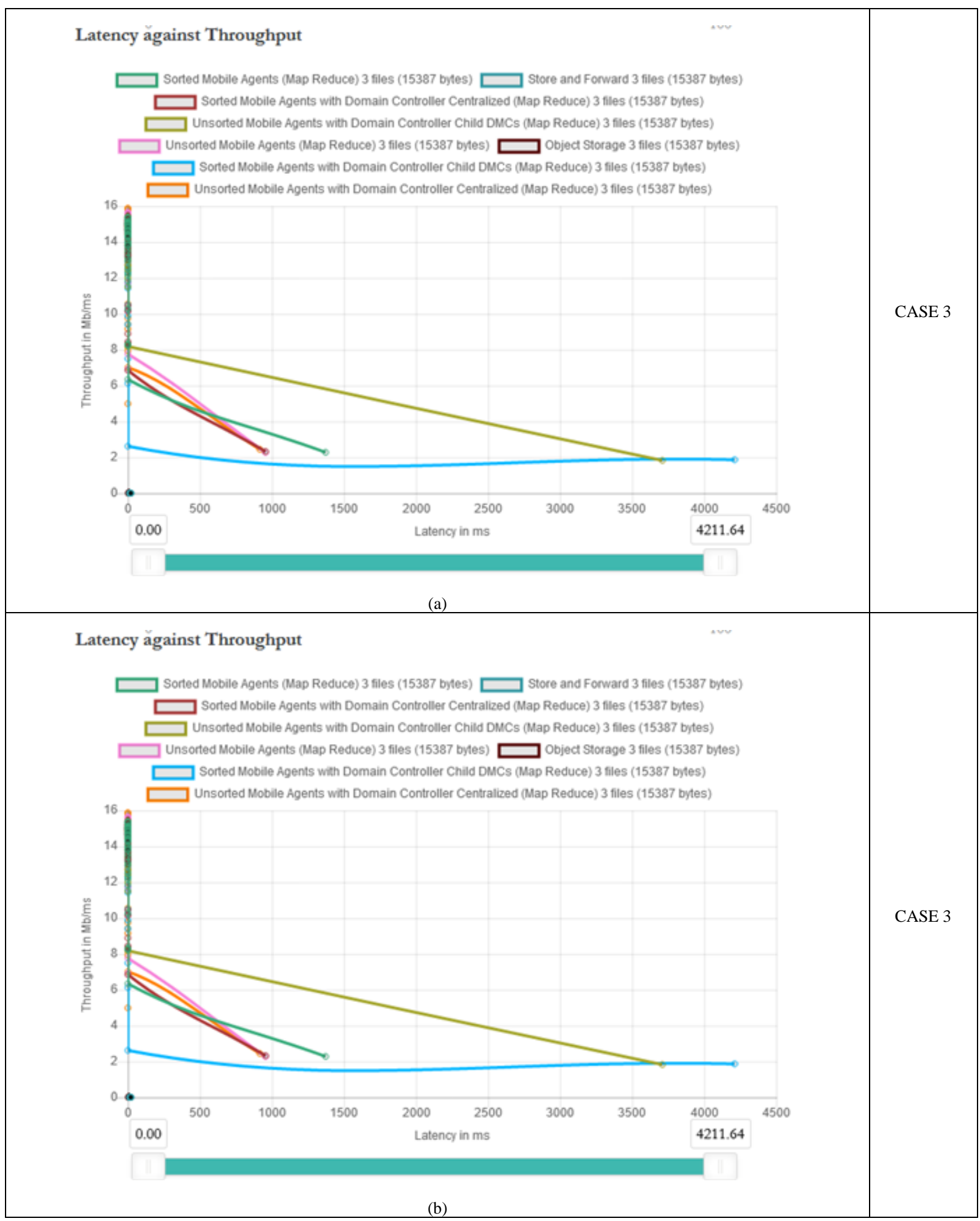




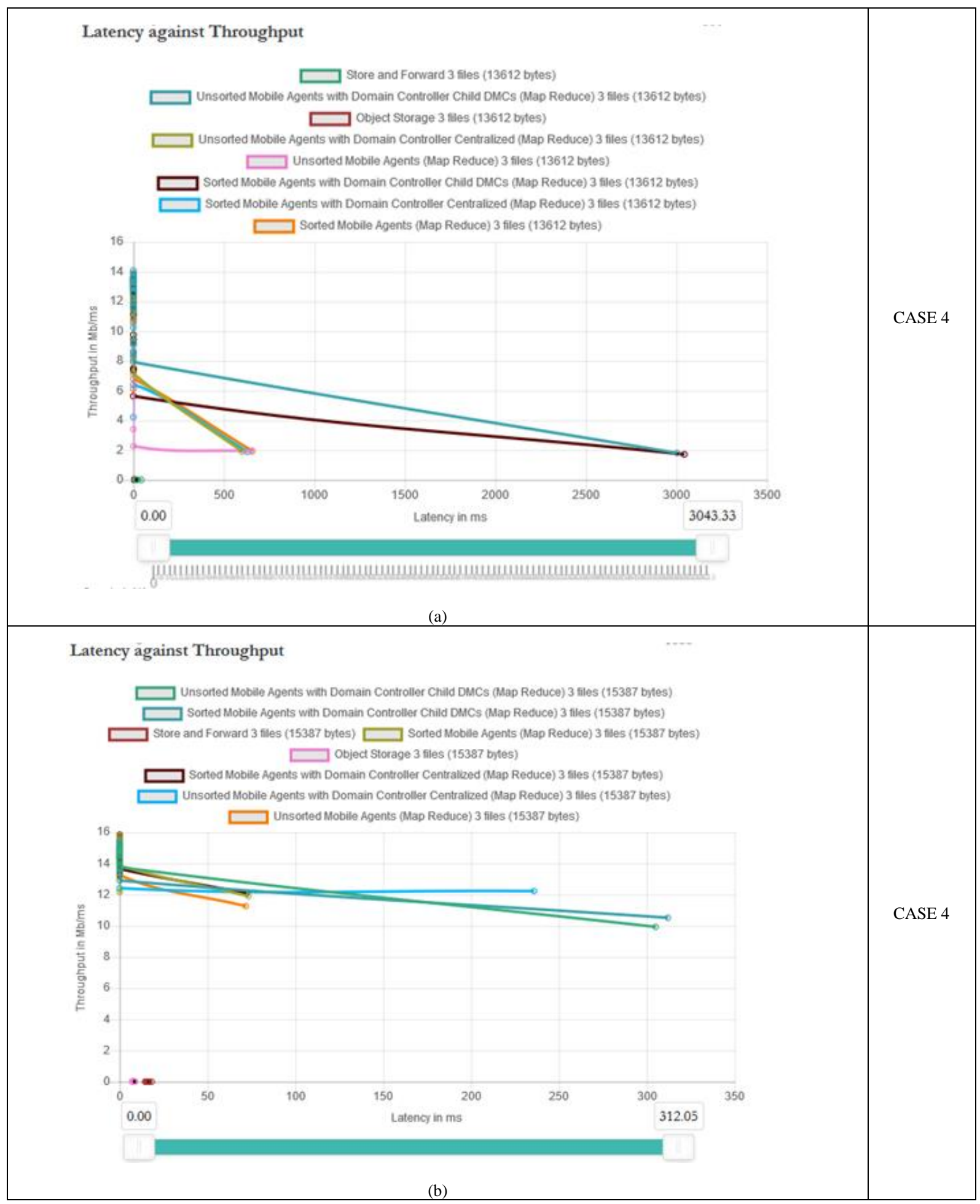

Table 5 above shows that latency decreases with increase in load for the agent based cases but remains at zero remains at the minimum for both OSD and SAF. Case 4a for instance indicates that at Zero Latency sorted mobile agents with domain controllers have the highest throughput and at $3000 \mathrm{~ms}$ latency throughput for sorted mobile agents with domain controller and sorted mobile agents with a centralized controller their throughput drastically reduced to 4 $\mathrm{Mb} / \mathrm{ms}$ from the initial $8 \mathrm{Mb} / \mathrm{ms}$. In $4 \mathrm{~b}$ at Zero Latency All mobile agents based metadata sorting with map reduce exhibit highest throughput at averagely $14 \mathrm{Mb} / \mathrm{ms}$ unlike for $4 \mathrm{a}$ where the cases averaged around $7 \mathrm{Mb} / \mathrm{ms}$ and also the average latency moves drastically from the high of 3000 to an average high of $300 \mathrm{~ms}$ a tenfold decrease in Latency. All the other cases exhibit similar characteristics. This shows that agents are better in terms of improving latencies and consequently increasing throughput even at high load capacities. 


\subsection{Scalability over Time}

The graphs in this table section represents how the system scales over time and it is subdivided into two columns sections a) and b): column a represents 100 client requests and column b) represents 1000 client requests.

Table 6. Scalability over time

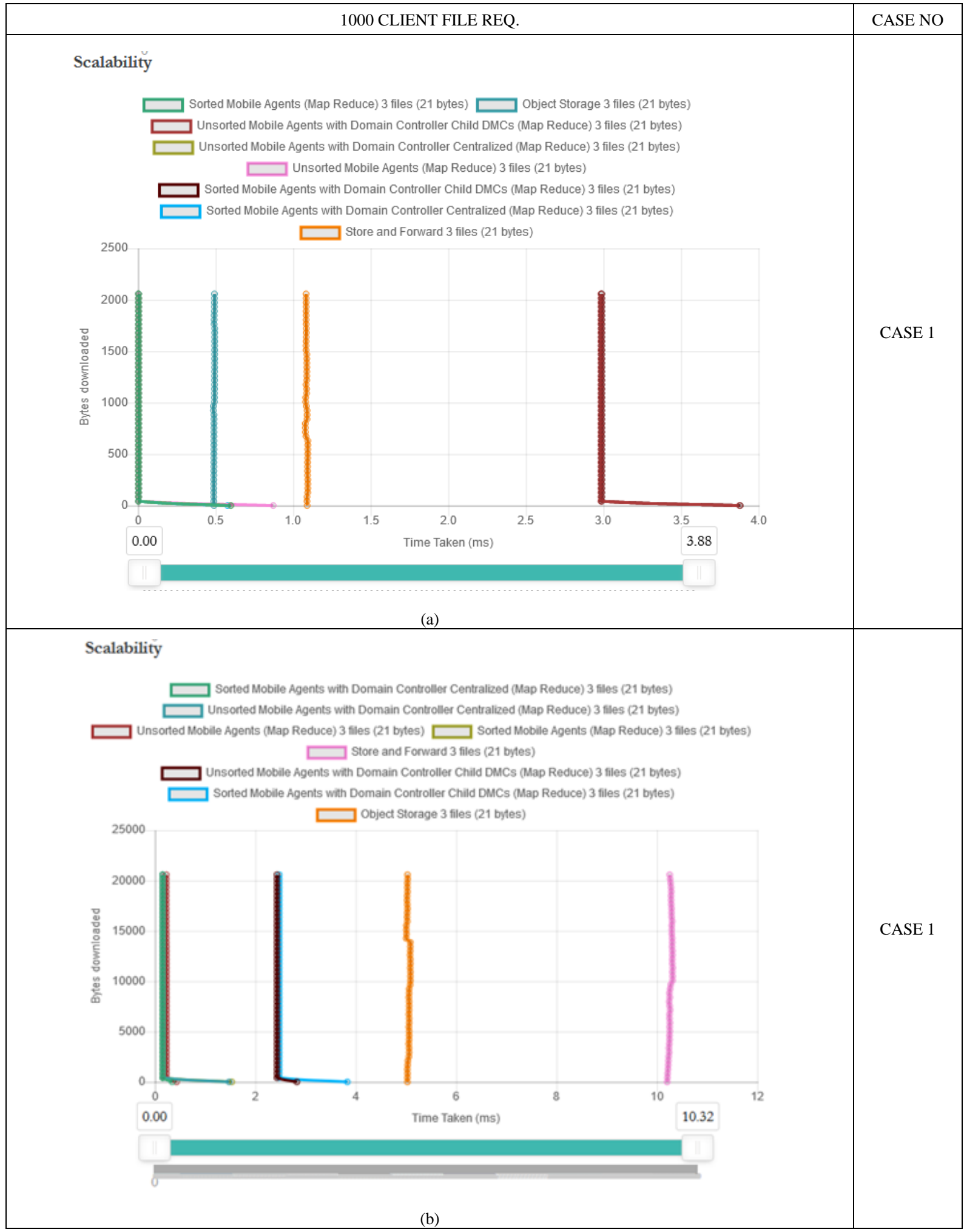




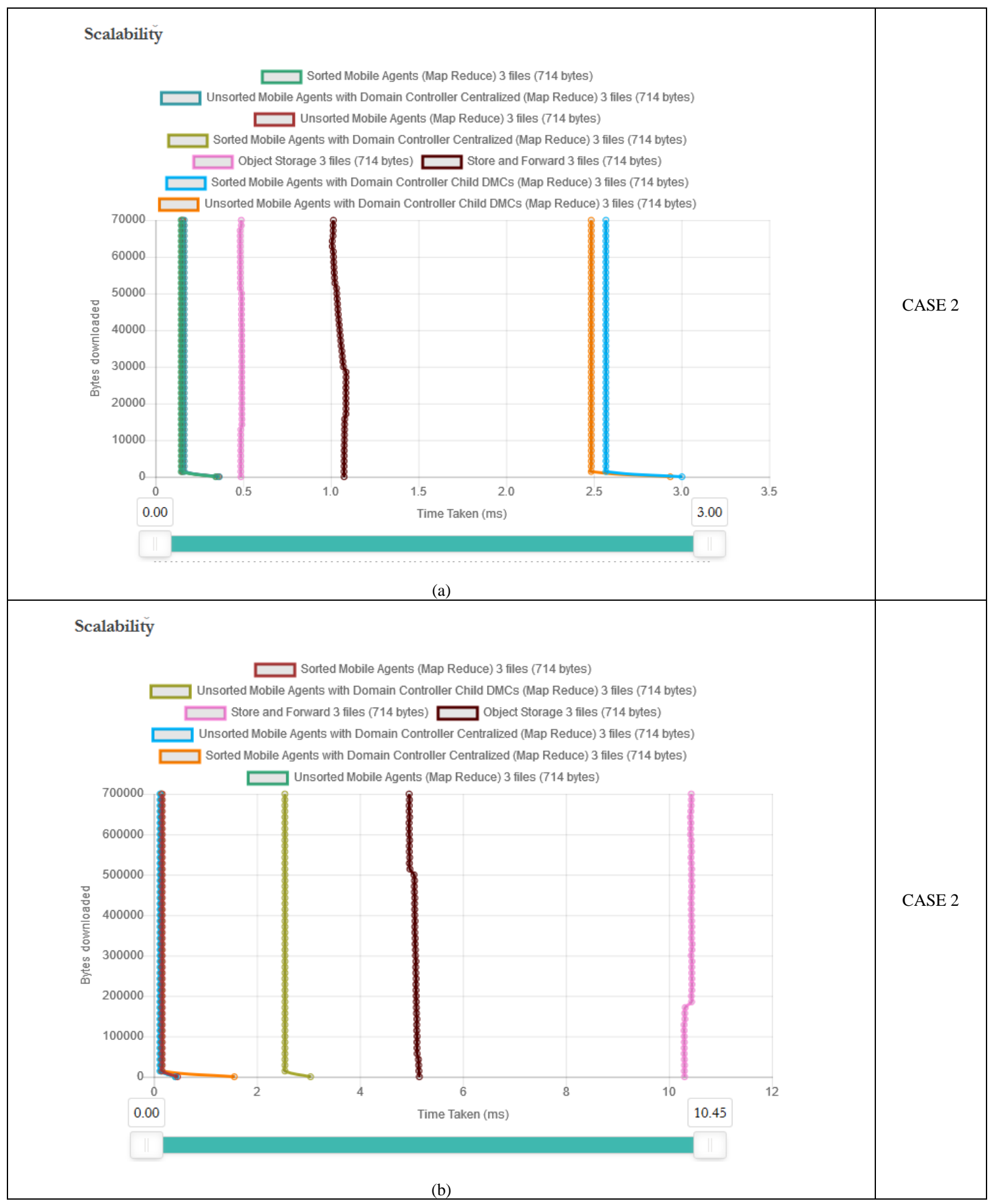




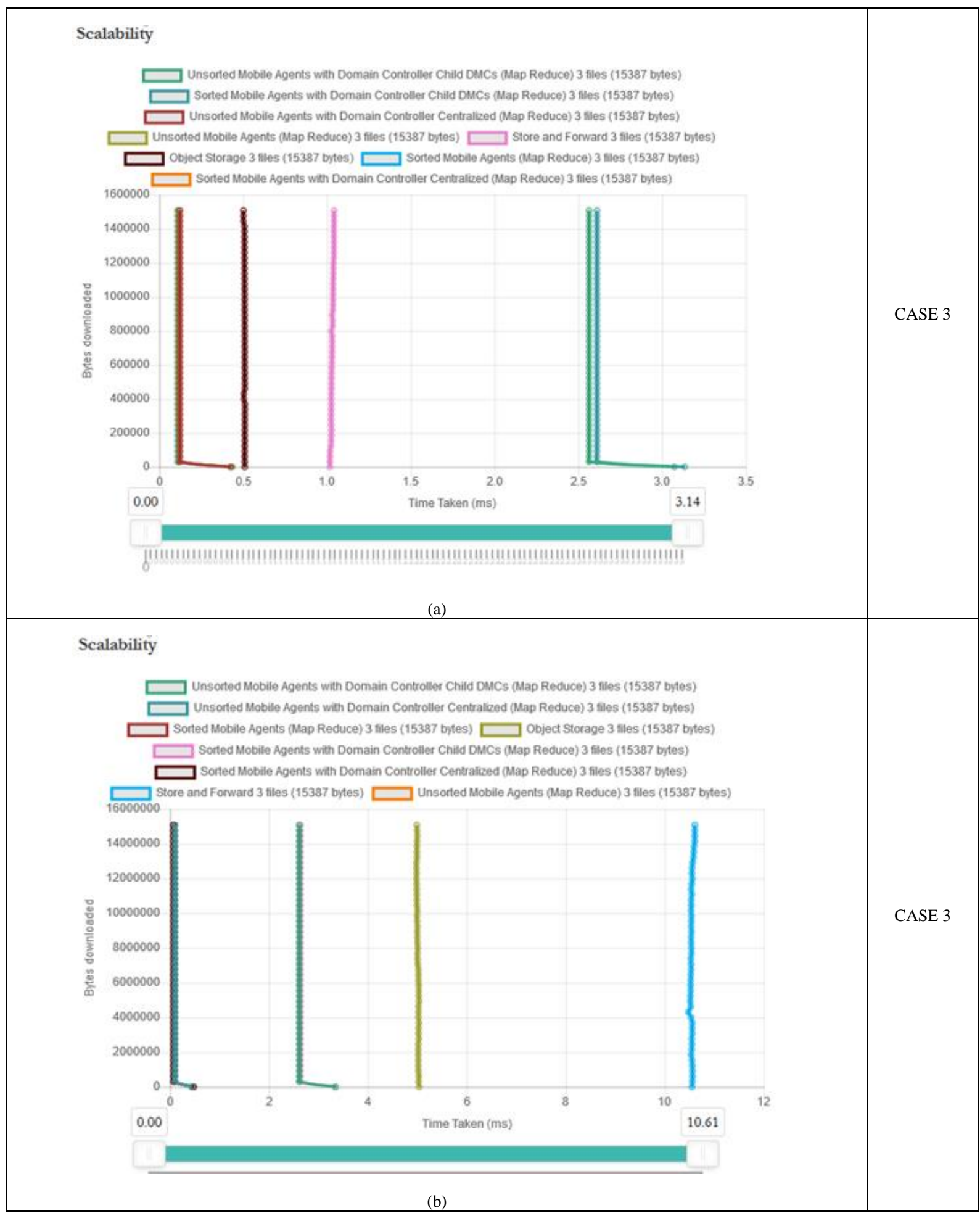




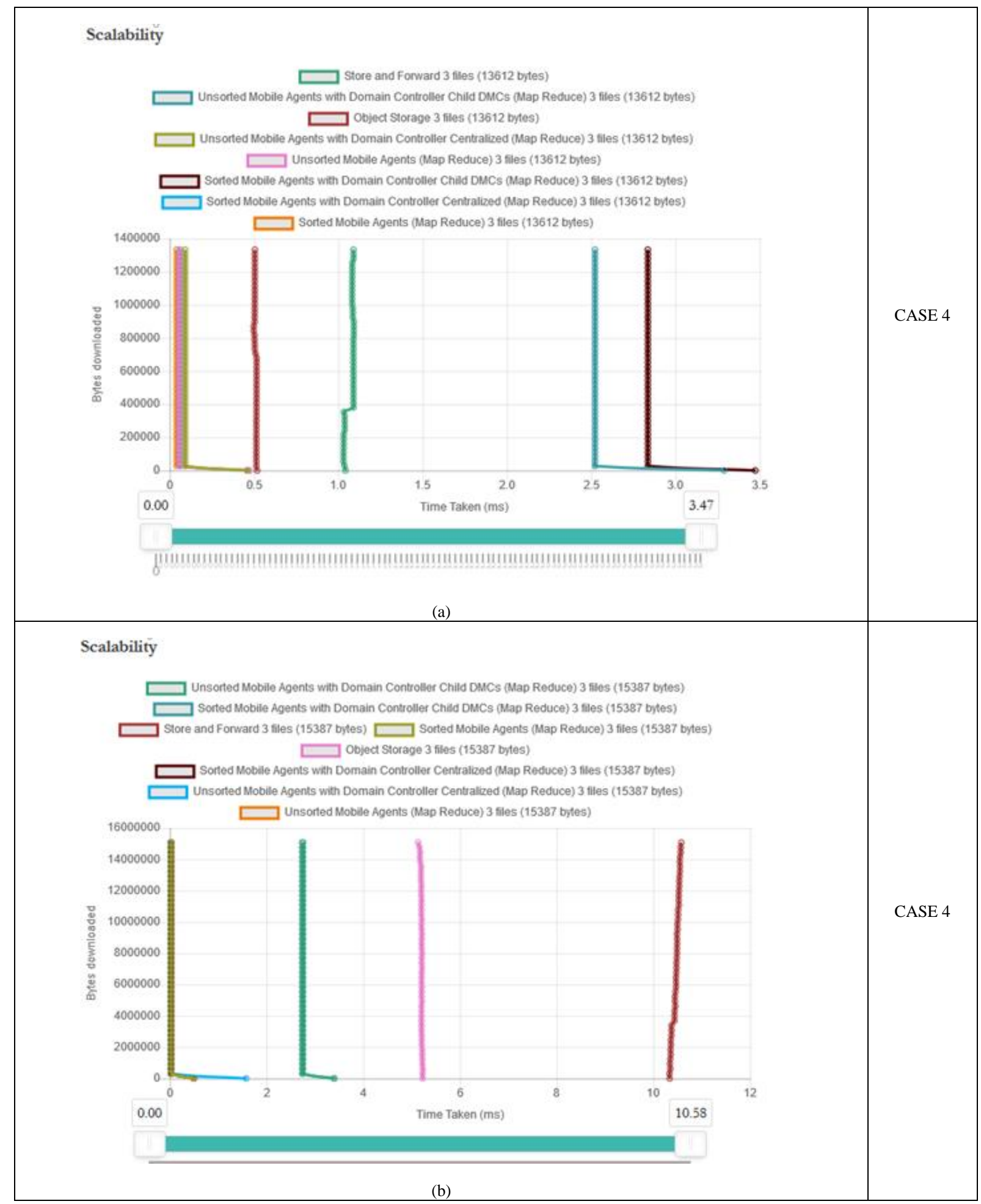


Table 6 above shows scalability of the SABSA engine with both 100 and 1000 client requests with files from three different SANs.

Table 6 above shows scalability over time, for instance CASE 4 shows that sorted agents with map reduce are the fastest in hundred client requests and sorted mobile agents with domain controllers don't perform well at this point on the other hand when the requests are increased to 1000 the sorted mobile agents with domain controllers have the best scalability, this shows that sorting of metadata by use of map reduce and further catching them improves not only performance but such systems are also highly scalable. Case 4a for instance shows that at an average of 1300000 bytes sorted mobile with child DMCs agents do not scale well at an average of $2.8 \mathrm{~ms}$ while unsorted mobile agents with map reduce without child DMCs perform well with the same load at $0 \mathrm{~ms}$. For case $4 \mathrm{~b}$ the at 15000000 bytes the unsorted mobile agents with map reduce still have the best scalability at $0 \mathrm{~ms}$ and store and forward has the worst scalability at averagely $10.5 \mathrm{~ms}$. But overall the salability sorted mobile with child DMCs agents improves significantly to average at $2.5 \mathrm{~ms}$.

\subsection{Case Summaries for Csv File Data}

The data in this section that was captured Table 7 to 12 below indicates a summary of the CSV outputs that were analyzed under various workload then Average time in Millisecond (ms), Throughput and latencies were captured and the compared under this predefined conditions.

It important to note that the files were run as batch files including multiple client requests for the required file in this experiment we have considered small and medium range workload requests.

A summarized table for the output of the time variance for downloading 1000 client requests from 100 client requests. 


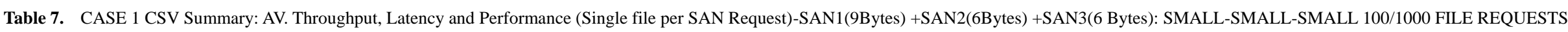

\begin{tabular}{|c|c|c|c|c|c|c|c|c|c|}
\hline Parameters & SAF & OSD & $\begin{array}{l}\text { Un-sorted } \\
\text { MA-MR }\end{array}$ & $\begin{array}{c}\text { Sorted } \\
\text { MA-MR }\end{array}$ & $\begin{array}{c}\text { Sorted } \\
\text { Centralized } \\
\text { MA-MR+DMC }\end{array}$ & $\begin{array}{c}\text { Unsorted } \\
\text { Centralized } \\
\text { MA-MR+DMC }\end{array}$ & $\begin{array}{c}\text { Sorted De-Centralized } \\
\text { MA-MR+DMC+Child } \\
\text { DMCs }\end{array}$ & $\begin{array}{c}\text { Unsorted } \\
\text { De-Centralized } \\
\text { MA-MR+DMC+ } \\
\text { Child DMCs } \\
\end{array}$ & $\begin{array}{c}\text { Total File } \\
\text { Size(SAN1+SAN2+S } \\
\text { AN3)(Bytes) }\end{array}$ \\
\hline AV.TT(ms) 1000 & 10.17 & 5.00 & 0.23 & 0.15 & 0.16 & 0.16 & 2.47 & 2.43 & $2.1 \times 10^{1}$ \\
\hline Av.TT(ms) 100 & 1.02 & 0.49 & 0.15 & 0.14 & 0.15 & 0.15 & 2.38 & 2.37 & $2.1 \times 10^{1}$ \\
\hline Throughput MB/s 1000 & $1.07 \times 10^{-5}$ & $2.21 \times 10^{-5}$ & 0.02 & 0.02 & 0.02 & 0.02 & 0.02 & 0.02 & $2.1 \times 10^{1}$ \\
\hline Throughput MB/s 100 & $1.06 \times 10^{-5}$ & $2.21 \times 10^{-5}$ & 0.02 & 0.02 & 0.02 & 0.12 & 0.02 & 0.02 & $2.1 \times 10^{1}$ \\
\hline AV.Latency(ms) 1000 & 15.17 & 7.48 & 0.23 & 0.15 & 0.81 & 4.21 & 7.81 & 4.84 & $2.1 \times 10^{1}$ \\
\hline AV.Latency(ms)100 & 15.21 & 7.37 & 8.12 & 7.88 & 7.76 & 7.77 & 46.71 & 46.39 & $2.1 \times 10^{1}$ \\
\hline
\end{tabular}

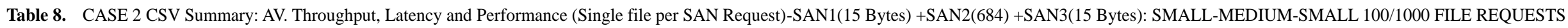

\begin{tabular}{|c|c|c|c|c|c|c|c|c|c|}
\hline Parameters & SAF & OSD & $\begin{array}{c}\text { Un-sorted } \\
\text { MA-MR }\end{array}$ & $\begin{array}{c}\text { Sorted } \\
\text { MA-MR }\end{array}$ & $\begin{array}{c}\text { Sorted } \\
\text { Centralized } \\
\text { MA-MR+DMC }\end{array}$ & $\begin{array}{c}\text { Unsorted } \\
\text { Centralized } \\
\text { MA-MR+DMC }\end{array}$ & $\begin{array}{c}\text { Sorted De-Centralized } \\
\text { MA-MR+DMC+Child } \\
\text { DMCs }\end{array}$ & $\begin{array}{c}\text { Unsorted } \\
\text { De-Centralized } \\
\text { MA-MR+DMC+ } \\
\text { Child DMCs } \\
\end{array}$ & $\begin{array}{l}\text { Total File Size } \\
\text { (Bytes) }\end{array}$ \\
\hline AV.TT(ms) 1000 & 10.02 & 4.92 & 0.17 & 0.17 & 0.17 & 0.18 & 2.15 & 2.18 & $7.14 \times 10^{2}$ \\
\hline Av.TT(ms) 100 & 1.04 & 0.49 & 0.16 & 0.15 & 0.15 & 2.49 & 2.57 & 2.49 & $7.14 \times 10^{2}$ \\
\hline Throughput MB/s 1000 & 0.00 & 0.00 & 0.65 & 0.66 & 0.66 & 0.67 & 0.66 & 0.67 & $7.14 \times 10^{2}$ \\
\hline Throughput MB/s 100 & 0.00 & 0.00 & 0.64 & 0.63 & 0.63 & 0.64 & 0.60 & 0.62 & $7.14 \times 10^{2}$ \\
\hline AV.Latency(ms) 1000 & 15.12 & 7.37 & 0.89 & 0.88 & 0.92 & 0.88 & 4.08 & 4.12 & $7.14 \times 10^{2}$ \\
\hline AV.Latency(ms)100 & 15.67 & 7.24 & 8.52 & 8.13 & 8.29 & 8.42 & 50.96 & 50.79 & $7.14 \times 10^{2}$ \\
\hline
\end{tabular}

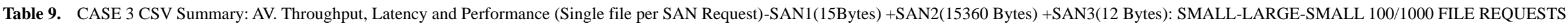

\begin{tabular}{|c|c|c|c|c|c|c|c|c|c|}
\hline Parameters & SAF & OSD & $\begin{array}{l}\text { Un-sorted } \\
\text { MA-MR }\end{array}$ & $\begin{array}{c}\text { Sorted } \\
\text { MA-MR }\end{array}$ & $\begin{array}{c}\text { Sorted } \\
\text { Centralized } \\
\text { MA-MR+DMC }\end{array}$ & $\begin{array}{c}\text { Unsorted } \\
\text { Centralized } \\
\text { MA-MR+DMC }\end{array}$ & $\begin{array}{c}\text { Sorted De-Centralized } \\
\text { MA-MR+DMC+Child } \\
\text { DMCs }\end{array}$ & $\begin{array}{c}\text { Unsorted } \\
\text { De-Centralized } \\
\text { MA-MR+DMC+Child } \\
\text { DMCs } \\
\end{array}$ & $\begin{array}{c}\text { Total File } \\
\text { Size } \\
\text { (Bytes) }\end{array}$ \\
\hline AV.TT(ms) 1000 & 10.45 & 4.96 & 0.10 & 0.10 & 0.07 & 0.10 & 2.63 & 2.61 & $1.54 \times 10^{4}$ \\
\hline Av.TT(ms) 100 & 1.02 & 0.51 & 0.11 & 0.11 & 0.12 & 0.13 & 2.62 & 2.57 & $1.54 \times 10^{4}$ \\
\hline Throughput MB/s 1000 & 0.01 & 0.02 & 14.28 & 14.13 & 14.31 & 14.13 & 14.32 & 14.42 & $1.54 \times 10^{4}$ \\
\hline Throughput MB/s 100 & 0.01 & 0.02 & 13.73 & 13.73 & 13.81 & 13.92 & 13.70 & 14.24 & $1.54 \times 10^{4}$ \\
\hline AV.Latency(ms) 1000 & 15.64 & 7.43 & 1.27 & 1.16 & 1.33 & 1.16 & 6.08 & 6.12 & $1.54 \times 10^{4}$ \\
\hline AV.Latency $(\mathrm{ms}) 100$ & 15.22 & 7.48 & 11.12 & 11.20 & 10.88 & 10.74 & 54.83 & 54.93 & $1.54 \times 10^{4}$ \\
\hline
\end{tabular}




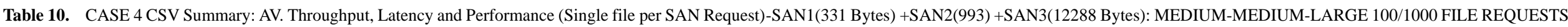

\begin{tabular}{|c|c|c|c|c|c|c|c|c|c|}
\hline Parameters & SAF & OSD & $\begin{array}{l}\text { Un-sorted } \\
\text { MA-MR }\end{array}$ & $\begin{array}{c}\text { Sorted } \\
\text { MA-MR }\end{array}$ & $\begin{array}{c}\text { Sorted } \\
\text { Centralized } \\
\text { MA-MR+DMC }\end{array}$ & $\begin{array}{c}\text { Unsorted } \\
\text { Centralized } \\
\text { MA-MR+DMC }\end{array}$ & $\begin{array}{c}\text { Sorted De-Centralized } \\
\text { MA-MR+DMC+Child } \\
\text { DMCs }\end{array}$ & $\begin{array}{c}\text { Unsorted } \\
\text { De-Centralized } \\
\text { MA-MR+DMC+ } \\
\text { Child DMCs } \\
\end{array}$ & $\begin{array}{c}\text { Total File Size } \\
\text { (Bytes) }\end{array}$ \\
\hline AV.TT(ms) 1000 & 10.37 & 5.16 & 0.02 & 0.02 & 0.03 & 0.02 & 2.74 & 2.76 & $1.36 \times 10^{4}$ \\
\hline Av.TT(ms) 100 & 1.07 & 0.51 & 0.06 & 0.05 & 0.07 & 0.10 & 2.84 & 2.53 & $1.36 \times 10^{4}$ \\
\hline Throughput MB/s 1000 & 0.01 & 0.02 & 14.50 & 14.51 & 14.64 & 14.34 & 14.65 & 14.41 & $1.36 \times 10^{4}$ \\
\hline Throughput MB/s 100 & 0.01 & 0.01 & 12.26 & 12.21 & 12.41 & 12.28 & 12.13 & 12.20 & $1.36 \times 10^{4}$ \\
\hline AV.Latency(ms) 1000 & 15.46 & 7.67 & 1.44 & 1.48 & 1.45 & 4.73 & 6.25 & 6.11 & $1.36 \times 10^{4}$ \\
\hline AV.Latency $(\mathrm{ms}) \mathbf{1 0 0}$ & 15.98 & 7.58 & 13.11 & 13.12 & 12.62 & 12.04 & 60.88 & 60.08 & $1.36 \times 10^{4}$ \\
\hline
\end{tabular}

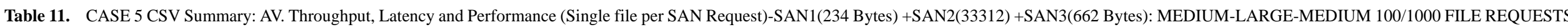

\begin{tabular}{|c|c|c|c|c|c|c|c|c|c|}
\hline Parameters & SAF & OSD & $\begin{array}{c}\text { Un-sorted } \\
\text { MA-MR }\end{array}$ & $\begin{array}{c}\text { Sorted } \\
\text { MA-MR }\end{array}$ & $\begin{array}{c}\text { Sorted } \\
\text { Centralized } \\
\text { MA-MR+DMC }\end{array}$ & $\begin{array}{c}\text { Unsorted } \\
\text { Centralized } \\
\text { MA-MR+DMC }\end{array}$ & $\begin{array}{c}\text { Sorted De-Centralized } \\
\text { MA-MR+DMC+Child } \\
\text { DMCs }\end{array}$ & $\begin{array}{c}\text { Unsorted } \\
\text { De-Centralized } \\
\text { MA-MR+DMC+ } \\
\text { Child DMCs } \\
\end{array}$ & $\begin{array}{c}\text { Total File Size } \\
\text { (Bytes) }\end{array}$ \\
\hline AV.TT(ms) 1000 & 10.57 & 5.20 & 0.01 & 0.01 & 0.01 & 0.01 & 2.86 & 2.86 & $3.42 \times 10^{4}$ \\
\hline Av.TT(ms) 100 & 1.06 & 0.50 & 0.04 & 0.01 & 0.01 & 0.02 & 2.57 & 2.60 & $3.42 \times 10^{4}$ \\
\hline Throughput MB/s 1000 & 0.01 & 0.01 & 13.41 & 13.63 & 13.37 & 13.53 & 13.39 & 13.69 & $3.42 \times 10^{4}$ \\
\hline Throughput MB/s 100 & 0.01 & 0.01 & 12.77 & 12.93 & 12.96 & 12.95 & 12.48 & 12.84 & $3.42 \times 10^{4}$ \\
\hline AV.Latency(ms) 1000 & 15.72 & 7.72 & 1.61 & 1.62 & 1.67 & 1.62 & 6.92 & 6.99 & $3.42 \times 10^{4}$ \\
\hline AV.Latency $(\mathrm{ms}) \mathbf{1 0 0}$ & 15.68 & 7.39 & 14.81 & 15.14 & 15.48 & 15.04 & 64.26 & 64.63 & $3.42 \times 10^{4}$ \\
\hline
\end{tabular}

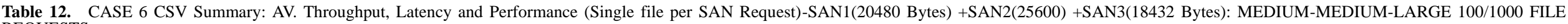
REQUESTS

\begin{tabular}{|c|c|c|c|c|c|c|c|c|c|}
\hline Parameters & SAF & OSD & $\begin{array}{c}\text { Un-sorted } \\
\text { MA-MR }\end{array}$ & $\begin{array}{c}\text { Sorted } \\
\text { MA-MR }\end{array}$ & $\begin{array}{c}\text { Sorted } \\
\text { Centralized } \\
\text { MA-MR+DMC }\end{array}$ & $\begin{array}{c}\text { Unsorted } \\
\text { Centralized } \\
\text { MA-MR+DMC }\end{array}$ & $\begin{array}{c}\text { Sorted } \\
\text { De-Centralized } \\
\text { MA-MR+DMC+ } \\
\text { Child DMCs } \\
\end{array}$ & $\begin{array}{c}\text { Unsorted } \\
\text { De-Centralized } \\
\text { MA-MR+DMC+Child } \\
\text { DMCs } \\
\end{array}$ & $\begin{array}{c}\text { Total File Size } \\
\text { (Bytes) }\end{array}$ \\
\hline AV.TT(ms) 1000 & 10.53 & 5.19 & 0.01 & 0.01 & 0.01 & 0.01 & 2.91 & 2.93 & $6.45 \times 10^{4}$ \\
\hline Av.TT(ms) 100 & 1.07 & 0.52 & 0.01 & 0.01 & 0.01 & 0.01 & 2.92 & 2.93 & $6.45 \times 10^{4}$ \\
\hline Throughput MB/s 1000 & 0.03 & 0.07 & 60.85 & 60.98 & 61.19 & 6.72 & 61.60 & 61.34 & $6.45 \times 10^{4}$ \\
\hline Throughput MB/s 100 & 0.03 & 0.07 & 58.32 & 58.20 & 56.91 & 57.38 & 57.29 & 57.47 & $6.45 \times 10^{4}$ \\
\hline AV.Latency $(\mathrm{ms}) 1000$ & 15.92 & 7.73 & 1.85 & 1.88 & 1.83 & 5.10 & 7.14 & 7.32 & $6.45 \times 10^{4}$ \\
\hline AV.Latency $(\mathrm{ms}) 100$ & 16.01 & 7.72 & 17.67 & 26.85 & 16.88 & 17.56 & 68.37 & 67.69 & $6.45 \times 10^{4}$ \\
\hline
\end{tabular}


5.5.1. Effect on Time Taken the Csv File Analysis

Table 13. Comparison of all the cases for the average time taken

\begin{tabular}{|c|c|c|c|c|c|c|c|c|c|}
\hline \multicolumn{10}{|c|}{ COMPARISON OF THE CASESFOR THE AVERAGE TME TAKEN } \\
\hline TYPE & SAF & OSD & Us-rerted & Surted & Surted Cosetralized & Uourerted Costrolized & Surted Do -Cosetralized & Uosserted Do-Cosetralized & AFERAGES \\
\hline & & & Ma MR & Ma MR & MA-MR+DHC & HA-MR+DHC & HA-HR+DHC+CLild DHG & HA-HR+DHC+CLild DHG & \\
\hline CASE 11100 & 1.02 & 0.49 & 0.15 & 0.14 & 0.15 & 0.15 & 2.38 & 2.37 & 6.85 \\
\hline CASE 11000 & 10.17 & 5.00 & 0.23 & 0.15 & 0.16 & 0.16 & 2.47 & 2.43 & 20.77 \\
\hline DIFF & 9.15 & 4.51 & 0.08 & 0.01 & 0.01 & 0.01 & 0.09 & 0.06 & 13.92 \\
\hline \%C1 Increased time & 89.71 & 92.04 & 5.33 & 0.71 & 0.67 & 0.67 & 0.38 & 0.25 & 20.32 \\
\hline CASE2H100 & 1.04 & 0.49 & 0.16 & 0.15 & 0.15 & 2.49 & 2.57 & 2.49 & 9.54 \\
\hline CASE2H1000 & 10.20 & 4.92 & 0.17 & 0.17 & 0.17 & 0.18 & 2.15 & 2.18 & 20.14 \\
\hline DIFF & 9.16 & 4.43 & 0.01 & 0.02 & 0.02 & .231 & .0 .42 & .0 .31 & 10.60 \\
\hline \% C2 Increased time & e 88.08 & 90.41 & 0.63 & 1.33 & 1.33 & -9.28 & -1.63 & -1.24 & 11.11 \\
\hline CASE 3100 & 10.45 & 0.51 & 0.11 & 0.11 & 0.12 & 0.13 & 2.62 & 2.57 & 16.62 \\
\hline CASE 31000 & 0.51 & 4.96 & 0.10 & 0.10 & 0.07 & 0.10 & 2.63 & 2.61 & 11.08 \\
\hline DIFF & 9.94 & .4 .45 & 0.01 & 0.01 & 0.05 & 0.03 & .0 .01 & .0 .04 & 5.54 \\
\hline \% C3 Increased time & 95.12 & -87.25 & 0.91 & 0.91 & 4.17 & 2.31 & -0.04 & -0.16 & 3.33 \\
\hline CASE 4100 & 1.07 & 0.51 & 0.06 & 0.05 & 0.07 & 0.10 & 2.84 & 2.53 & 7.23 \\
\hline CASE 41000 & 10.37 & 5.16 & 0.02 & 0.02 & 0.03 & 0.02 & 2.74 & 2.76 & 21.12 \\
\hline DIFF & 9.30 & 4.65 & .0 .04 & .0 .03 & .0 .04 & .0 .08 & .0 .10 & 0.23 & 13.89 \\
\hline \% C4 Increased time & 86.92 & 91.18 & -6.67 & -6.00 & -5.71 & -8.00 & -0.35 & 0.91 & 19.21 \\
\hline CASE 5100 & 1.06 & 0.50 & 0.01 & 0.01 & 0.01 & 0.02 & 2.57 & 2.60 & 6.78 \\
\hline CASE 5H1000 & 10.57 & 5.20 & 0.04 & 0.01 & 0.01 & 0.01 & 2.86 & 2.86 & 21.56 \\
\hline DIFF & 9.51 & 4.70 & 0.03 & 0.00 & 0.00 & .0 .01 & 0.29 & 0.26 & 14.78 \\
\hline \% C5 Increased time & 897.17 & 94.00 & 30.00 & 0.00 & 0.00 & -5.00 & 1.13 & 1.00 & 21.80 \\
\hline CASE BH100 & 1.07 & 0.52 & 0.01 & 0.01 & 0.01 & 0.01 & 2.92 & 2.93 & 7.48 \\
\hline CASE 6 H1000 & 10.53 & 5.19 & 0.01 & 0.01 & 0.01 & 0.01 & 2.91 & 2.93 & 21.60 \\
\hline DIFF & 9.46 & 4.67 & 0.00 & 0.00 & 0.00 & 0.00 & .0 .01 & 0.00 & 14.12 \\
\hline \% C6 Increased time & 88.41 & 89.81 & 0.00 & 0.00 & 0.00 & 0.00 & -0.03 & 0.00 & 18.88 \\
\hline Overall aw\% increase in tim & 20.93 & 4.64 & 0.41 & -0.06 & 0.00 & -0.33 & -0.02 & 0.01 & 1.24 \\
\hline
\end{tabular}

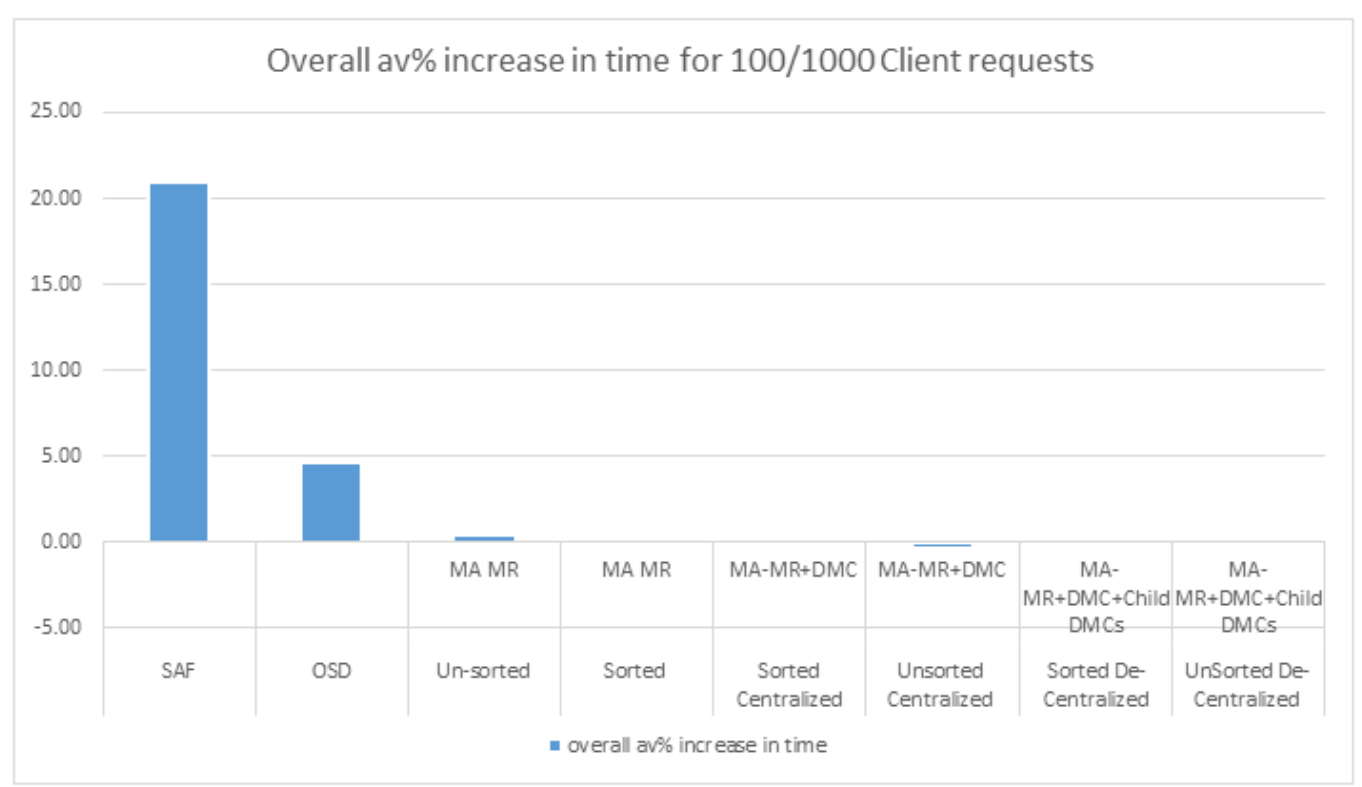

Figure 4. Bar charts showing average increase in time for 100 and 1000 client requests

Figure 4 above shows a summary of the overall individual percentage averages,for Case 1 to Case 6 , of how the time is affected when the client requests increased from 100 the initial number of requests to 1000 requests for each identified method in the SABSA engine. SAF has the largest time difference at at $20.9 \%$ more time followed by OSD at $4.64 \%$, but the agent based and map reduce based objects have insgnificant change in time in servicing this request. 
5.5.2. Effect on Latencies for the Csv File Analysis

5.5.2.1. Summary Latencies Analysis

Table 14. Comparison of all the cases for the latencies

\begin{tabular}{|c|c|c|c|c|c|c|c|c|c|c|}
\hline TYPE & SAF & OSD & Un-sorte & Sorted & Sorted C & Unsorted & Sorted D & UnSort & AVERAGES & \\
\hline & & & MA MR & MA MR & MA-MR- & MA-MR+ & MA-MR & MA-MF & -DMC+Child & DMCs \\
\hline CASE $1 / 100$ & 15.21 & 7.37 & 0.15 & 7.88 & 7.76 & 7.77 & 46.71 & 46.39 & 139.24 & \\
\hline CASE $1 / 1000$ & 15.17 & 7.48 & 0.23 & 0.15 & 0.81 & 4.21 & 7.81 & 4.84 & 40.70 & \\
\hline DIFF & -0.04 & 0.11 & 0.08 & -7.73 & -6.95 & -3.56 & -38.90 & -41.55 & -98.54 & \\
\hline$\% \mathrm{Cl}$ Increased time & -0.26 & 1.49 & 53.33 & -98.10 & -89.56 & -45.82 & -83.28 & -89.57 & -70.77 & \\
\hline CASE $2 / 100$ & 15.67 & 7.24 & 8.52 & 8.13 & 8.29 & 8.42 & 50.96 & 50.79 & 158.02 & \\
\hline CASE $2 / 1000$ & 15.12 & 7.37 & 0.89 & 0.88 & 0.92 & 0.88 & 4.08 & 4.12 & 34.26 & \\
\hline DIFF & -0.55 & 0.13 & -7.63 & -7.25 & -7.37 & -7.54 & -46.88 & -46.67 & -123.76 & \\
\hline$\%$ C2 Decrease Latencies & -3.51 & 1.80 & -89.55 & -89.18 & -88.90 & -89.55 & -91.99 & -91.89 & -78.32 & \\
\hline CASE $3 / 100$ & 15.22 & 7.48 & 11.12 & 11.20 & 10.88 & 10.74 & 54.83 & 54.93 & 176.40 & \\
\hline CASE $3 / 1000$ & 15.64 & 7.43 & 1.27 & 1.16 & 1.33 & 1.16 & 6.08 & 6.12 & 40.19 & \\
\hline DIFF & -0.42 & 0.05 & 9.85 & 10.04 & 9.55 & 9.58 & 48.75 & 48.81 & 136.21 & \\
\hline \% C3 Decrease Latencies & -2.76 & 0.67 & 88.58 & 89.64 & 87.78 & 89.20 & 88.91 & 88.86 & 77.22 & \\
\hline CASE $4 / 100$ & 15.98 & 7.58 & 13.11 & 13.12 & 12.62 & 12.04 & 60.88 & 60.08 & 195.41 & \\
\hline CASE $4 / 1000$ & 15.46 & 7.67 & 1.44 & 1.48 & 1.45 & 4.73 & 6.25 & 6.11 & 44.59 & \\
\hline DIFF & -0.52 & 0.09 & -11.67 & -11.64 & -11.17 & -7.31 & -54.63 & -53.97 & -150.82 & \\
\hline \% C4 Decrease Latencies & -3.25 & 1.19 & -89.02 & -88.72 & -88.51 & -60.71 & -89.73 & -89.83 & -77.18 & \\
\hline CASE $5 / 100$ & 15.68 & 7.39 & 14.81 & 15.14 & 15.48 & 15.04 & 64.26 & 64.63 & 212.43 & \\
\hline CASE $5 / 1000$ & 15.72 & 7.72 & 1.61 & 1.62 & 1.67 & 1.62 & 6.92 & 6.99 & 43.87 & \\
\hline DIFF & 0.04 & 0.33 & -13.20 & -13.52 & -13.81 & -13.42 & -57.34 & -57.64 & -168.56 & \\
\hline$\%$ C5 Decrease Latencies & 0.26 & 4.47 & -89.13 & -89.30 & -89.21 & -89.23 & -89.23 & -89.18 & -79.35 & \\
\hline CASE $6 / 100$ & 16.01 & 7.72 & 17.67 & 26.85 & 16.88 & 17.56 & 68.37 & 67.69 & 238.75 & \\
\hline CASE $6 / 1000$ & 15.92 & 7.73 & 1.85 & 1.88 & 1.83 & 5.10 & 7.14 & 7.32 & 48.77 & \\
\hline DIFF & -0.09 & 0.01 & -15.82 & -24.97 & -15.05 & -12.46 & -61.23 & -60.37 & -189.98 & \\
\hline \% C6 Decrease Latencies & -0.56 & 0.13 & -89.53 & -93.00 & -89.16 & -70.96 & -89.56 & -89.19 & -79.57 & \\
\hline overall av\% increase in Laten & -1.64 & 1.37 & -44.78 & -45.09 & -44.67 & -36.87 & -45.27 & -45.21 & -39.53 & \\
\hline
\end{tabular}

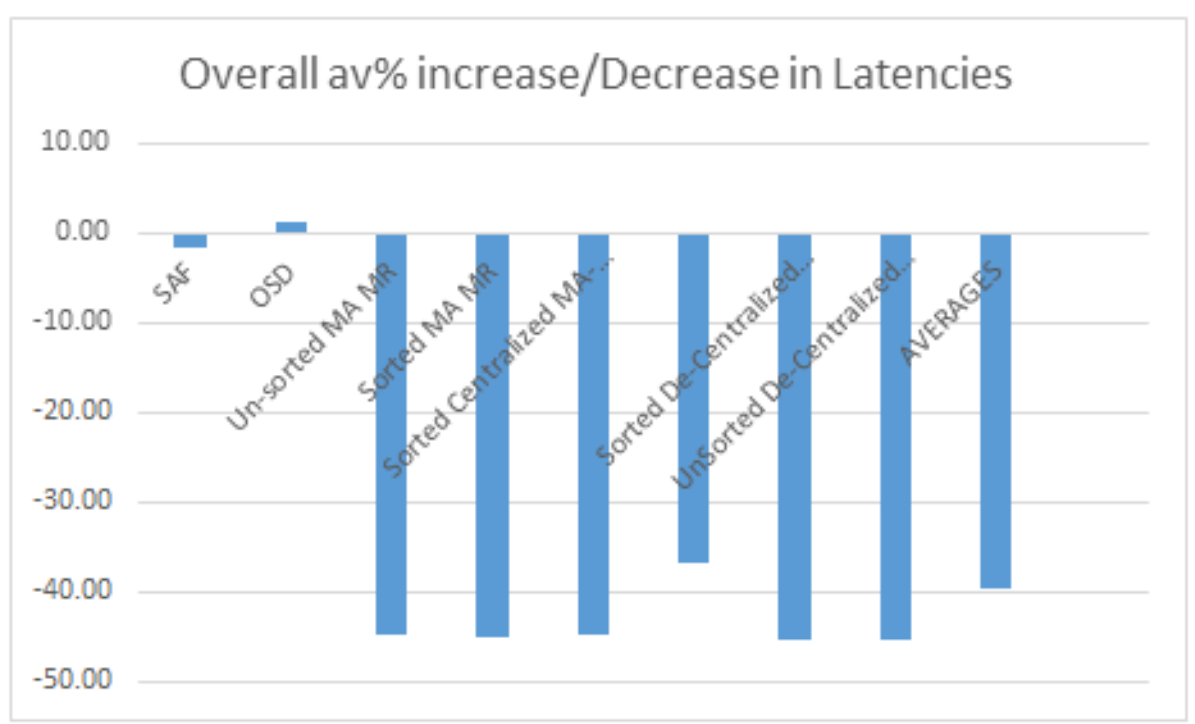

Figure 5. Bar charts showing average increase/decrease in overall latencies for 100/1000 client requests

Figure 5 above shows a bar chart for the latencies. The outputs show both positive and negative outputs. The negative ouputs indicate better utilization of the system by minimizing latencies which are a penalty to system performance. The positive values signify increase in latencies which impedes system perfomance. The margins above are also represented as percentage reduction of the overall considered methods in Figure 5 above.

This section has demonstrated various scenarios of viewing the data generated by the SABSA engine using the bar graphs and pie charts and line graphs; various cases generated by the decision tree were generated and captured into the decision matrix where a few random cases were chosen, indicated as Case 1-Case 6 to demonstrate the performance of the SABSA Engine under various load capacities. 


\subsubsection{Effect on Throughput}

5.5.3.1. Summary of Throughput Analysis

Table 15. Comparison of all the cases for the throughput

\begin{tabular}{|c|c|c|c|c|c|c|c|c|c|}
\hline \multicolumn{10}{|c|}{\begin{tabular}{l|l|l|l|} 
COMPARISON OF THE CASES FOR THE AVERAGE THROUGHPUTS & & & \\
\end{tabular}} \\
\hline & & \begin{tabular}{l|l|l|l|} 
COMPARISON OF THE CASES FOR THE AVERAGE THROOGHPOTS \\
TYPR & SAF & OSD & In-sorted
\end{tabular} & & \multicolumn{5}{|c|}{ Sorted Cent Unsorted Ce Sorted De-C UnSorted De AVERAGES } \\
\hline & & & & \begin{tabular}{|l|} 
Sorted \\
MA MR \\
\end{tabular} & \multicolumn{2}{|c|}{ MA-MR+DMMA-IR+DM } & \multicolumn{3}{|c|}{ MA-MR+DMMA-MR+DMC+Child DMC } \\
\hline CASE $1 /$ & & & & & & & & & \\
\hline CASE $1 / 1000$ & & & & & & & & & \\
\hline \multirow{2}{*}{$\begin{array}{l}\text { DIFF } \\
\text { \% Cl Increased Throghput }\end{array}$} & & & & & & & & & \\
\hline & & & & & & & & & \\
\hline \multirow{2}{*}{$\frac{\text { CASE } 2 / 100}{\text { CASE } 2 / 1000}$} & & & & & & & & & \\
\hline & & & & & & & & & \\
\hline \multirow{2}{*}{$\begin{array}{l}\text { DIFF } \\
\% \text { C2 Increased Throghput }\end{array}$} & & & & & & & & & \\
\hline & & & & & & & & & \\
\hline \multirow{2}{*}{$\begin{array}{l}\text { CASE } 3 / 100 \\
\text { CASE } 3 / 1000\end{array}$} & & & & & & & & & \\
\hline & & & & & & & & & \\
\hline \multirow{2}{*}{$\begin{array}{l}\text { DIFF } \\
\text { \% C3 Increased Throghput }\end{array}$} & & & & & & & & & \\
\hline & & & & & & & & & \\
\hline \multirow{2}{*}{$\begin{array}{l}\text { CASE 4/100 } \\
\text { CASE 4/1000 }\end{array}$} & & & & & & & & & \\
\hline & & & & & & & & & \\
\hline \multirow{2}{*}{$\begin{array}{l}\text { DIFF } \\
\text { \% C4 Increased Throghput }\end{array}$} & & & & & & & & & \\
\hline & & 00000 & & & & & & & \\
\hline CASE $5 / 100$ & & & & & & & & & \\
\hline CASE 5/1000 & & & & & & & & & \\
\hline \multirow{2}{*}{$\begin{array}{l}\text { DIFF } \\
\text { \% C5 Increased Throghput }\end{array}$} & & & & & & & & & \\
\hline & & & & & & & & & \\
\hline CASE 6/100 & & & & & & & & 57.4700000 & 345.6700000 \\
\hline CASE 6/1000 & & & & & & & & 61.3400000 & 312.7800000 \\
\hline \multirow{2}{*}{$\begin{array}{l}\text { DIFF } \\
\text { \% C6 Increased Throghput }\end{array}$} & & & & & & & & & \\
\hline & & & & & & & & & \\
\hline overall av\% increase in Latenct & 0.0000000 & 16.6666667 & 4.1962256 & 5.1459990 & 4.9658244 & -10.6426190 & 6.8440309 & 6.3781852 & 2.8145519 \\
\hline
\end{tabular}

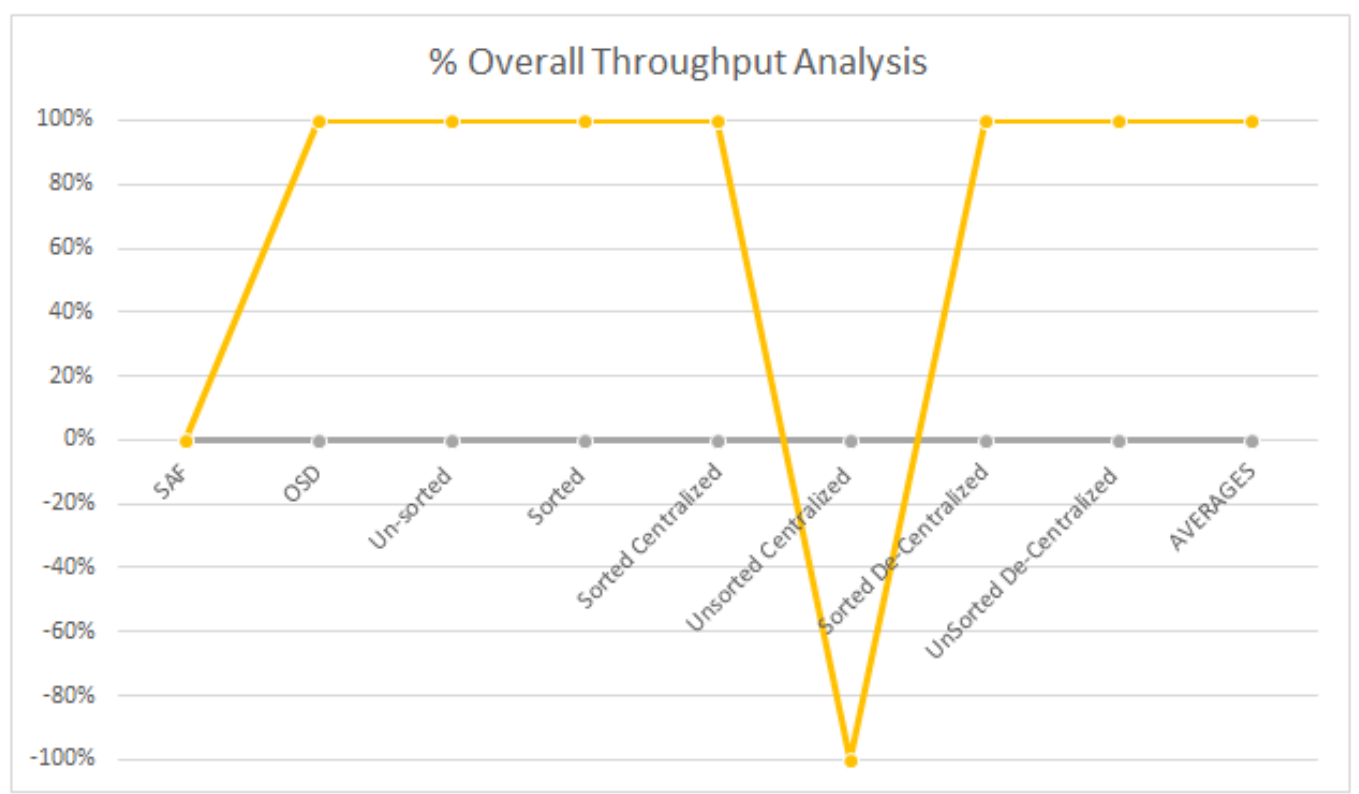

Figure 6. A Line graph showing average overall throuput 100-1000 client requests 
Figure 6 is a summary Line graph of the Comparison of all the cases for the throughput in table 15 above ; it shows that store and forward (SAF) has the lowest throughput at $0 \%$ and sorting of metadata sorting of metadata and introducing an agent also has a positive impact in increasing throughput of a system performing at maximum throughput of $100 \%$ for OSD and all agent based methods except the sorted decentralized whose throughput drastically drops and then resume back to maximum throughput after some time.

\section{Conclusions and Further Work}

As observed from the previous analysis, all cases indicate that sorting of metadata and caching it will make this system faster than their counter-parts with centralized metadata. In conclusion our experiments tend to concur with Amidal's law that splitting a system into sub systems improves the performance of such a system up to a certain limit.

Mobile agents play a key role in contributing to the performance improvement of the distributed system environment as has been indicated in the previous observations. Mobile agents can autonomously move from one place to another with metadata and security being guaranteed. This research question led to discovery of new tools like SPADE framework for agent design within the python programming environment this greatly contributed to implementation of mobile agents in with the Docker containers.

Since mobile agents is a new concept, and virtualization and big data are emerging trends, this technology will be important in defining and re-defining such research directions. Mobile agents can also be applied in the study of Internet of things that greatly relies on virtualization technologies and therefore offload the virtual server from the mundane work of security, load balancing and job tracking.

Map reduce help improve locality of reference of the metadata functions with Key, value pairs for target storage domains being shuffled together and then the agent caches this sorted metadata domains which consequently leads to shorter access paths and thus minimization of latencies and consequently increasing performance of such systems.

Further work will be carried as follows:

1) Extend the concept of Mobile Agent (MA) based virtualization to the field of Artificial Intelligence (AI) and Artificial Neural networks (ANNs); in order to solve the exponential data requirements in the IOT systems. ANNs will provide multiple distributed nodes that will communicate with other peers within a given domain and eventually transfer the processed data the distributed virtual child nodes or domains to the parent Nodes/Domains and eventually to the parent nodes/domain for final storage or processing.
2) To improve on our SABSA Test-Bed simulator to a more advanced simulator with an adaptable API-to allow for testing of applications-; including organizations for testing their storage requirements in regards to: scalability, Latencies and throughput.

3) To study and implement advanced security fencing systems within our agent-based storage architecture.

\section{REFERENCES}

[1] Al-shishtawy, A. (2012) Self-Management for Large-Scale Distributed Systems.

[2] Alberola, J. M. et al. (2010) 'A performance evaluation of three Multiagent Platforms', Artificial Intelligence Review, 34(2), pp. 145-176. doi: 10.1007/s10462-010-9167-9.

[3] Amazon (2019) 10-Minute Tutorials. Available at: https://aws.amazon.com/getting-started/tutorials/.

[4] Andrei, P. S. et al. (2014) 'Evolution towards Distributed Storage in a Nutshell', pp. 1267-1274.

[5] Anon (2016) Concordia White paper. Available at: https://www.cis.upenn.edu/bcpierce/629/papers/Concordia -Whitepaper/ (Accessed: 17 March 2016).

[6] Arias (2018) Introduction to Redis:Installation,CLI commands and Data-Types.

[7] Avilés-González, A., Piernas, J. and González-Férez, P. (2014) 'Scalable metadata management through OSD+ devices', International Journal of Parallel Programming, 42(1), pp. 4-29. doi: 10.1007/s10766-012-0207-8.

[8] Banks, C. M. (no date) 'Principles of Modeling and Simulation: A Multidisciplinary Approach'.

[9] Caidi, M. et al. (2008) 'The Google File System Sanjay', Journal de Chirurgie, 145(3), pp. 298-299. doi: 10.1016/S0021-7697(08)73776-1.

[10] Ceph (2016) Welcome to Ceph. Available at: http://docs.ceph.com/docs/master/\# (Accessed: 30 April 2019).

[11] Chaturvedi, V. (no date) Deep Dive into Docker. Available at: https://www.edureka.co/blog/what-is-docker-container (Accessed: 26 March 2019).

[12] CORP (2016) Content addressed storage systems, EMC. Available at: http:www.emc.com/products/systems/centera .jsp?openfolder=platform (Accessed: 26 June 2016).

[13] Docker (2019) Docker Docs. Available at: https://docs.docker.com/v17.09/compose/install/ (Accessed: 27 March 2019).

[14] EMC2 (2008) Where information lives:current benefit and future potential technology concepts and business considerations.

[15] Escriv, M., C, J. P. and Bada, G. A. (2014) 'A Jabber-based Multi-Agent System Platform *', (January 2006). doi: 10.1145/1160633.1160866. 
[16] Escriva, R. and Wong, B. (no date) 'Http://Hyperdex.Org/Papers/Hyperdex.Pdf', Hyperdex. Org. Available at: http://hyperdex.org/papers/hyperdex.pdf \%5Cnpapers2://publication/uuid/7E524955-B159-492D-B 9E4-F52C5E1BAE79.

[17] Factor, M. et al. (2006) 'Object Storage: The Future Building Block for Storage Systems A Position Paper’, pp. 119-123. doi: 10.1109/lgdi.2005.1612479.

[18] Feng, D. et al. (2004) 'Enlarge Bandwidth of Multimedia Server with Network Attached Storage System 3 The Redirection of Data Transfer', pp. 489-492.

[19] Finin, T. (1992) An Overview of KQML: A Knowledge Query and Manipulation Language.

[20] FIPA (2000) 'Foundation for Intelligent Physical Agents', Inform.

[21] FIPA (2002) 'FIPA Abstract Architecture Specification (SC00001L)', p. 75.

[22] FullStack (no date) Full Stack Python,Redis. Available at: https://www.fullstackpython.com/redis.html (Accessed: 28 March 2019).

[23] Gibson, G. A. et al. (2001) 'A cost-effective, high-bandwidth storage architecture', High Performance Mass Storage and Parallel I/O: Technologies and Applications, (May 2014), pp. 431-444. doi: 10.1109/9780470544839.ch28.

[24] Griffit (2018) how-build-hello-redis-with-python, Opensource.com.

[25] Hanemann, A. et al. (2006) 'A study on network performance metrics and their composition', Campus-Wide Information Systems, 23(4), pp. 268-282. doi: $10.1108 / 10650740610704135$.

[26] Hendricks, J. et al. (2006) 'Improving small file performance in object-based storage’, (May).

[27] Hitachi (2016) Storage virtualisation:How to capitalize on its economic benefits.

[28] Iii, W. B. L. and Ross, R. B. (2000) '4th Annual Linux Showcase \& Conference, Atlanta PVFS: A Parallel File System for Linux Clusters $£$ '.

[29] James (2006) 'Improving small file performance in object based storage.', CMU-PDL-06-104.

[30] James, J. (no date) "“Cassandra", Notes and Queries, s2-X(241), p. 111. doi: 10.1093/nq/s2-X.241.111-a.

[31] Kabir, M. H. et al. (2014) 'Detail Comparison of Network Simulators', (November). doi: 10.13140/RG.2.1.3040.912 8.

[32] Kang, S. J., Lee, S. Y. and Lee, K. M. (2015) 'Performance Comparison of OpenMP, MPI, and MapReduce in Practical Problems', Advances in Multimedia, 2015, pp. 1-9. doi: $10.1155 / 2015 / 575687$

[33] Karakoyunlu, C. et al. (2013) 'Toward a Unified Object Storage Foundation for Scalable Storage Systems'.

[34] Kasireddy, P. (2016) A Beginner-Friendly Introduction to Containers, VMs and Docker. Available at: https://medium .freecodecamp.org/a-beginner-friendly-introduction-to-con tainers-vms-and-docker-79a9e3e119b?gi=a26c3acc92c1

(Accessed: 26 March 2018).

[35] Kaur, K. and Rai, A. K. (2014) 'A Comparative Analysis : Grid, Cluster and Cloud Computing', 3(3), pp. 5730-5734.

[36] Lange, D. B. (1998) 'Mobile objects and mobile agents: The future of distributed computing?', Lecture Notes in Computer Science (including subseries Lecture Notes in Artificial Intelligence and Lecture Notes in Bioinformatics), 1445, pp. 1-12. doi: 10.1007/BFb0054084.

[37] Lehner, W. (2013) Web-Scale Data Management for the Cloud.

[38] Li, G. et al. (2006) 'Researches on Performance Optimization of Distributed Integrated System Based on Mobile Agent*', pp. 4038-4041.

[39] Liancheng, X. U. (2014) 'Research on Distributed Data Stream Mining in Internet of Things', (Lemcs).

[40] Liu, X. et al. (2015) 'Meta-MapReduce for scalable data mining', Journal of Big Data. Journal of Big Data. doi: 10.1186/s40537-015-0021-4.

[41] Luck, M., McBurney, P. and Preist, C. (2003) 'Agent Technology: Enabling Next Generation Computing (A Roadmap for Agent Based Computing)'. Available at: http://eprints.soton.ac.uk/257309/.

[42] Maitrey, S. (2015) 'Handling Big Data Efficiently by using Map Reduce Technique'. doi: 10.1109/CICT.2015.140.

[43] Mark et al. (2000) Storage Virtualisation, What is it all about?

[44] McCanne, S., Vetterli, M. and Jacobson, V. (1997) 'Low-complexity video coding for receiver-driven layered multicast', IEEE Journal on Selected Areas in Communications, 15(6), pp. 983-1001. doi: 10.1109/49.61 1154.

[45] Mesnier, M. et al. (2003) ‘01222722’, (August), pp. 84-90.

[46] Microsystems, S. (2007) 'LUSTRE TM FILE SYSTEM', (December).

[47] Miller, E. L., Freeman, W. E. and Reed, B. C. (2002) 'Proceedings of the FAST 2002 Conference on File and Storage Technologies Strong Security for Network-Attached Storage', Access. Available at: http://www.usenix.org.

[48] Mishra, A. (2012) 'Application of Mobile Agent in Distributed Network Management'. doi: 10.1109/CSNT.20 12.198 .

[49] Mohammed, E. A., Far, B. H. and Naugler, C. (2014) 'Applications of the MapReduce programming framework to clinical big data analysis : current landscape and future trends', 7(1), pp. 1-23. doi: 10.1186/1756-0381-7-22.

[50] Moniem, H. A. and Ammar, H. H. (2015) 'A framework for Performance Prediction of Service-Oriented Architecture', International Journal of Computer Applications Technology and Research, 4(11), pp. 865-870. doi: 10.7753/ijcatr0411.1013.

[51] 'MSST-Cabrera' (1991) 'A storage Architecture for large objects'. 
[52] Mwathi, D. G. (2018) 'A model based approach for implimenting Authentication and access control in public WLANS: A CASE OF UNIVERSITIES IN KENYA', Director CSI, 15(2), pp. 2017-2019. doi: 10.22201/fq.1870 8404e.2004.3.66178

[53] Oracle, S. (2011) 'Lustre Software Release 2. x Operation Manual'.

[54] Osero, B. O. (2010) Storage virtualisation and management. University of Nairobi.

[55] Osero, B. O. (2013) 'NETWORK STORAGE VIRTUALISATION AND MANAGEMENT BENARD ONG ' ERA OSERO LECTURER Network Attached Devices , Storage virtualization , Security .', International Journal of Education and Research, 1(12), pp. 1-10.

[56] Oussous, A. et al. (2009) 'Comparison and Classification of NoSQL Databases for Big Data'.

[57] Outcomes, L. (no date) 'Understanding research philosophies and approaches', pp. 2-30.

[58] Palanca, J. (2018) 'SPADE Documentation'.

[59] Panasas, I. (2016) 'Panasas', Wikipedia. Available at: https://en.wikipedia.org/wiki/Panasas.

[60] Patel, A. B., Birla, M. and Nair, U. (2012) 'Addressing Big Data Problem Using Hadoop and Map Reduce’, pp. 6-8.

[61] Pedro Jos'e Marr 'on, Stamatis Karnouskos, D. M. A. O. and the C. consortium (2011) No Title.

[62] Permabit 'Permabit', (2015), Wikipedia. Available at: https://en.wikipedia.org/wiki/.

[63] Rajguru, P. (2011) 'Available Online at www.jgrcs.info ANALYSIS OF MOBILE AGENT', Journal of Global Research in Computer Science, 2(11), pp. 6-10. Available at: www.jgres.info.

[64] Randy, Fellows, A. R. and Kerns, R. (2012) 'SAN Virtualization Evaluation Guide', p. 2.

[65] Rfc, T., Rfc, T., et al. (no date) 'Ethernet RFC-2544 expained', pp. 1-9.

[66] Rfc, T., Engineering, I., et al. (no date) 'The RFC 2544 Application - Performance Benchmarking for the HaulPass V60s Link', pp. 1-11.

[67] Riedel, E. and Nagle, D. (1999) 'Active Disks - Remote Execution for Network-Attached Storage Thesis Committee':, Science, (December). Available at: https://p dfs.semanticscholar.org/74ac/0dd0a14ea27f016b170a1254 c14fe8c73b37.pdf.

[68] Rodríguez-enríquez, L. R. C. et al. (2015) 'A general perspective of Big Data: applications, tools', The Journal of Supercomputing. Springer US. doi: 10.1007/s11227-015 $-1501-1$

[69] Rugg, G. and Petre, M. (2004) 'The Unwritten Rules of PhD research', Open University Press, p. 241.

[70] Sargent, R. G. (2011) 'Advanced Tutorials: Verification and Validation of Simulation Models', Proceedings of the 2011 Winter Simulation Conference, pp. 183-198.

[71] Sarkar, N. I., Member, S. and Halim, S. A. (2011) ‘A Review of Simulation of Telecommunication Networks : Simulators, Classification, Comparison, Methodologies, and Recommendations'.

[72] Satoh, I. (2003) 'Building reusable mobile agents for network management', IEEE Transactions on Systems, Man and Cybernetics Part C: Applications and Reviews, 33(3), pp. 350-357. doi: 10.1109/TSMCC.2003.818944.

[73] Satoh, I. (2004) 'Dynamic Federation of Partitioned Applications', pp. 2-6.

[74] Satoh, I. (2011) 'Mobile Agent Middleware for Dependable Distributed Systems'.

[75] Satoh, I. (2014) 'MapReduce-based Data Processing on IoT', (iThings). doi: 10.1109/iThings.2014.32.

[76] Satoh, I. (2016) 'Agent-based MapReduce Processing in IoT', 1(Icaart), pp. 250-257. doi: 10.5220/0005802102500 257.

[77] Satoh, I. and Society, I. C. (2003) 'A Testing Framework for Mobile Computing Software’, 29(12), pp. 1112-1121.

[78] Silva, L. M. (1999) 'Optimizing the Migration of Mobile Agents'.

[79] Singavarapu, S. and Hariri, S. (2001) 'S ELF-MANAGING STORAGE SYSTEM - D ESIGN AND EVALUATION 2. Self Managing Storage System (SMSS) Architecture Overview'.

[80] Smith, R. (1999) '3ULQFLSOHV RI 0RGHOLQJ Fundamental Principles of Model Abstraction', pp. 1-28.

[81] Sowmya, N., Aparna, M. and Tijare, P. (2015) 'An Adaptive Load Balancing Strategy in Cloud Computing based on Map Reduce', (September), pp. 4-5

[82] Tate, J. et al. (2017) 'Introduction to Storage Area'.

[83] Tekniska, K., Ögskolan, H. and Simsarian, K. T. (2000) 'VETENSKAP OCH KONST Dissertation, March 2000 Computational Vision and Active Perception Laboratory (CVAP)', (March).

[84] Tutorialpoint (no date) REDIS - QUICK GUIDE REDIS ENVIRONMENT REDIS - DATA TYPES.

[85] Wakefield, R. (2007) 'An Analysis of Quality of Service Metrics and Frameworks in a Grid Computing Environment'.

[86] Wang, J. et al. (2010) 'A Novel Weighted-Graph-Based Grouping Algorithm for Metadata Prefetching A Novel Weighted-Graph-Based Grouping Algorithm for Metadata Prefetching'.

[87] Weil, S. A., Brandt, S. A. and Miller, E. L. (2006) 'CRUSH : Controlled, Scalable, Decentralized Placement of Replicated Data', (November).

[88] Welch, B. et al. (2008) 'White Paper Scalable Performance of the Panasas Parallel File System', Fast 2008, (May), pp. $1-22$.

[89] Wetoyi, A. O. (2014) 'UNIVERSITY OF NAIROBI Dynamic Subset Difference Revocation using One Binary Tree AUTHOR Austin Owino Wetoyi Prof William Okelo-Odongo', (December). 
[90] Wikipedia (2019) 'Network Performance', Wikipedia. Available at: https://en.wikipedia.org/wiki/Network_perfor mance.

[91] Wu, S. A. I. (2014) 'Distributed Data Management Using MapReduce', 46(3).

[92] Xu, H. and Shatz, S. M. (2001) 'A Design Model for Intelligent Mobile Agent Software Systems', pp. 1-23. Available at: file:///C:/Users/ltturche/Downloads/32bfe512 24d170bc42.pdf.

[93] Yazdi, H. T., Fard, A. M. and Akbarzadeh-T, M. R. (2008) 'Cooperative criminal face recognition in distributed web environment', AICCSA 08 - 6th IEEE/ACS International Conference on Computer Systems and Applications, (March), pp. 524-529. doi: 10.1109/AICCSA.2008.44935 82.

[94] $\mathrm{Yu}$, P. et al. (2006) 'Mobile Agent Enabled Application Mobility for', pp. 648-657. 